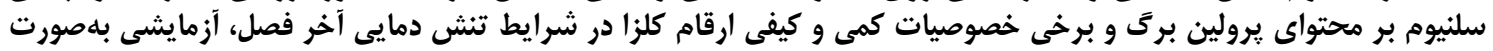

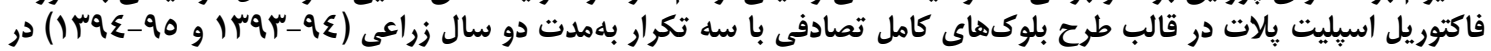

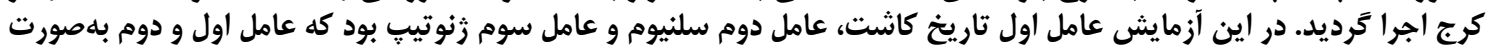

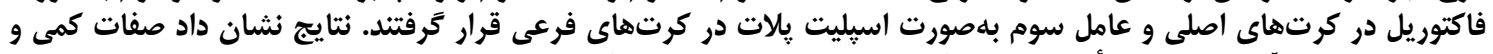

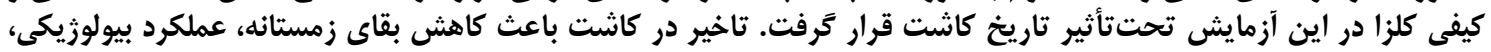

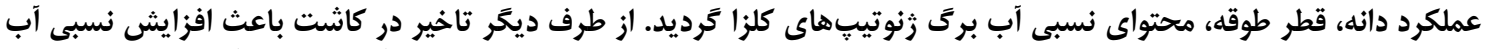

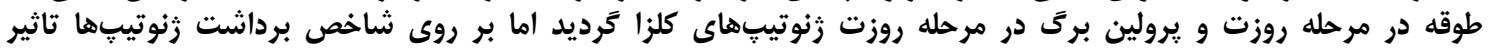

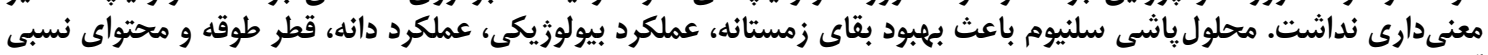

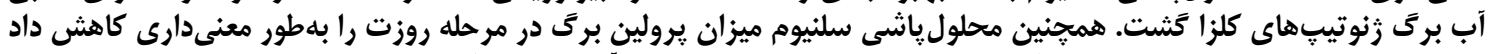

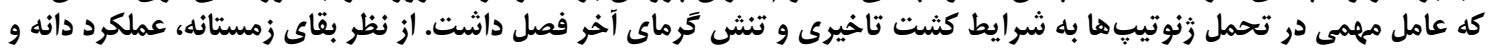

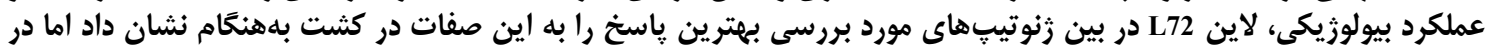

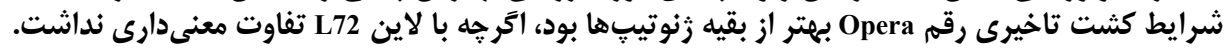

وازههاى كليدى: تاريخ كاشت، يرولين، عملكرد، زنوتيڤ، سلنات سديم

زمانى مراحل فنولوزيكى كَياه با عوامل محيطى مؤثر بر آنها

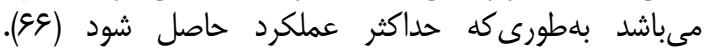

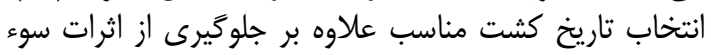

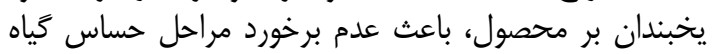

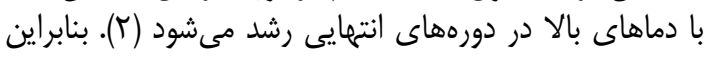

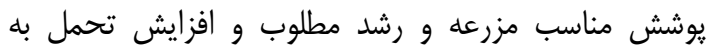

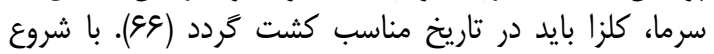

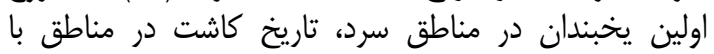

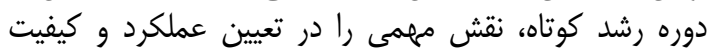

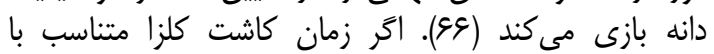

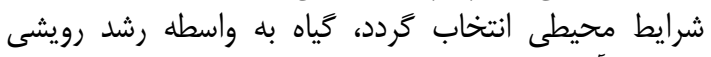

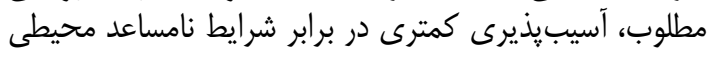

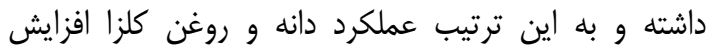

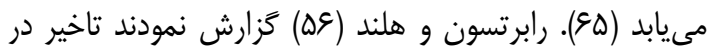

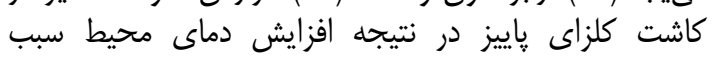

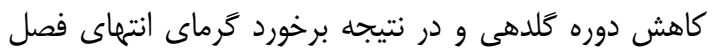

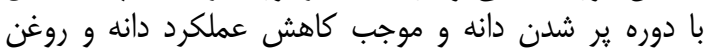

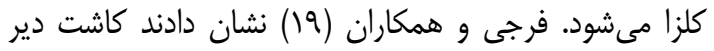

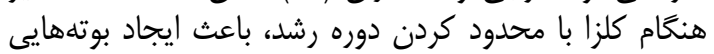

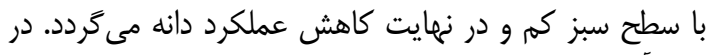

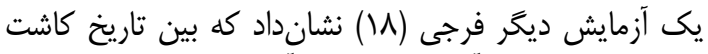

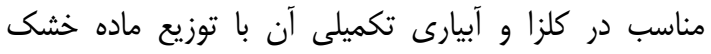

مقدمه

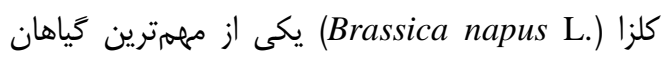

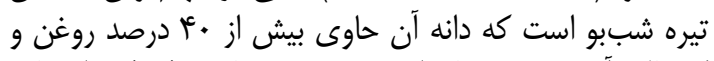

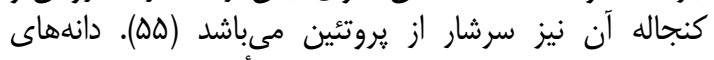

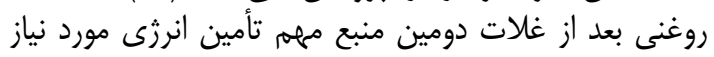

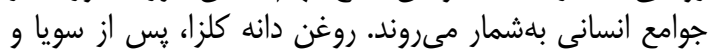

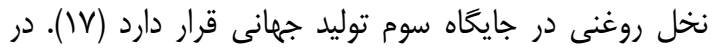

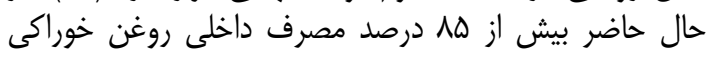

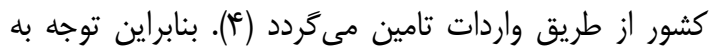

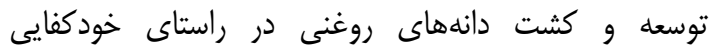
اقتصادى و تامين امنيت غذايى از الهميت زياني زيادى برخوردار

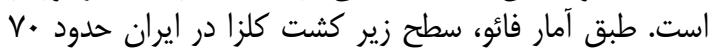

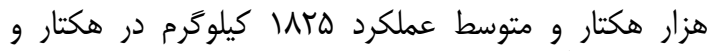

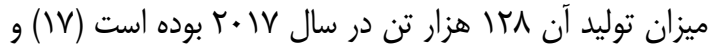

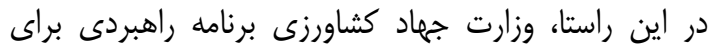

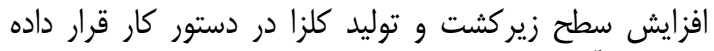

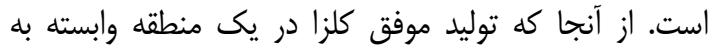

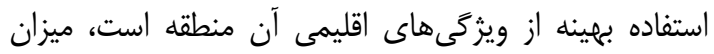

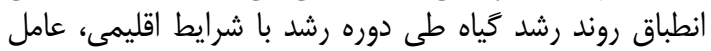

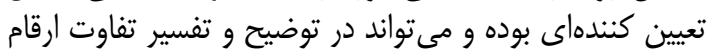

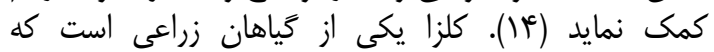

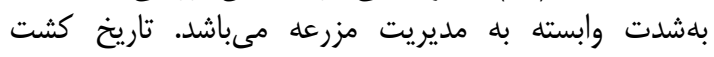
مناسب، ابزار مديريتى مهمى براى تعيين بهترين تطابق تاريخ كنيت 
بلميزان مقاومت كياه بستخى دارد. زنده ماندن كياهان ديان در

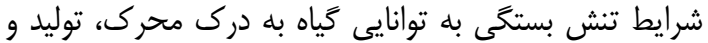

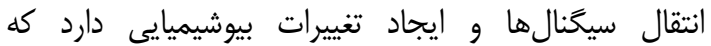

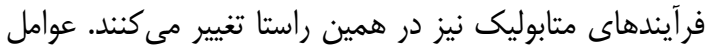

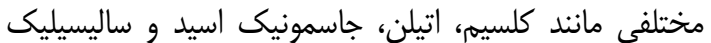

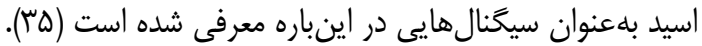

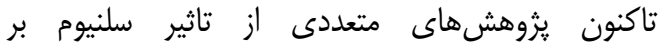

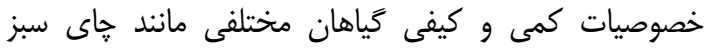

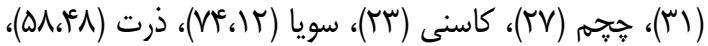

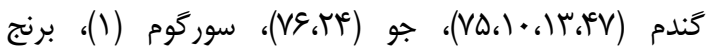

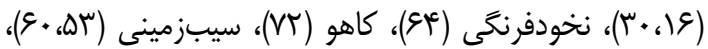

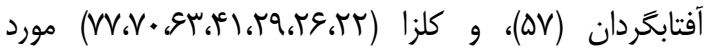

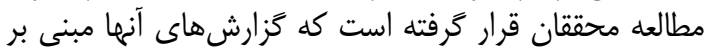

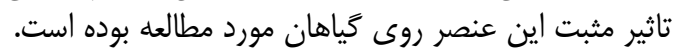

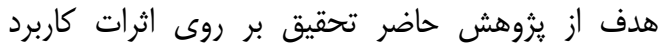

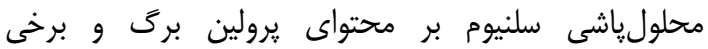

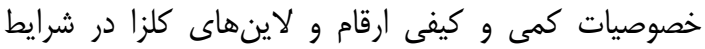

تنش گَرماى آخر فصل در تاريخهاى مختلف كاشت بودان

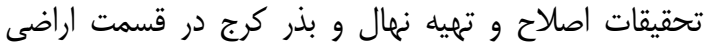

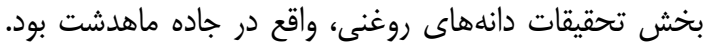

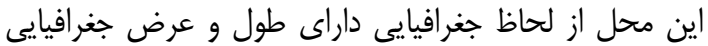

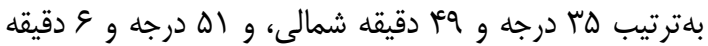

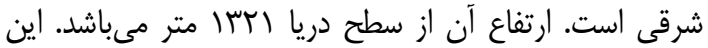

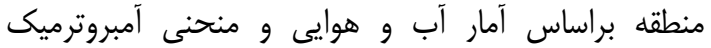

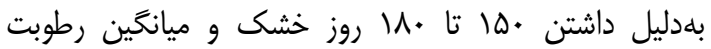

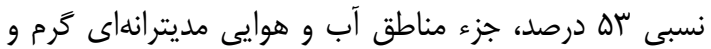

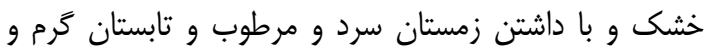

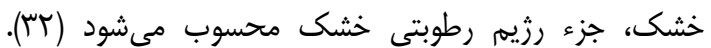

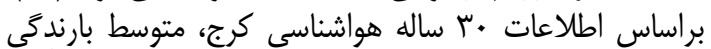

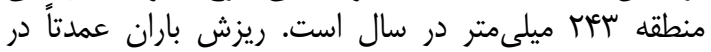

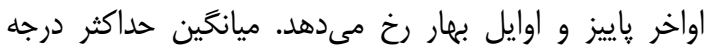

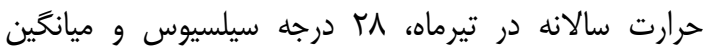

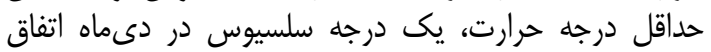

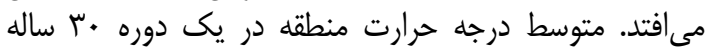

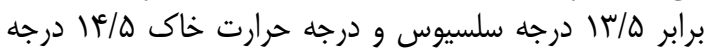

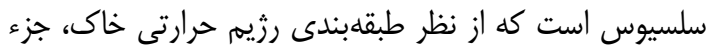

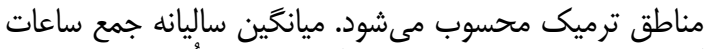

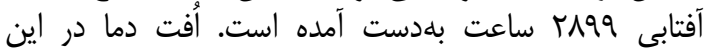

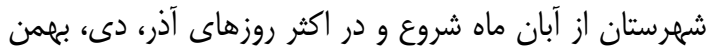

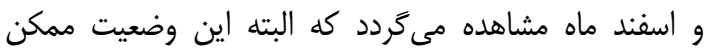

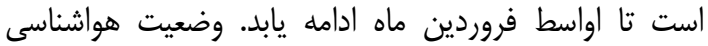

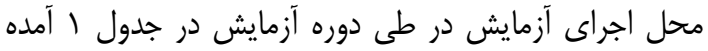

ارتباط مستقيمى وجود دارد كه از آن مىتوان در مدلسازى كياهى استفاده نمود.

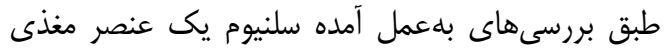

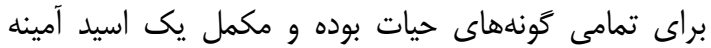

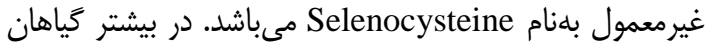

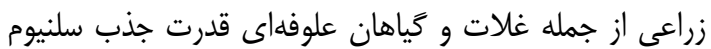

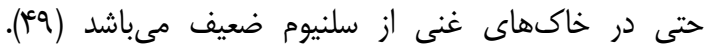

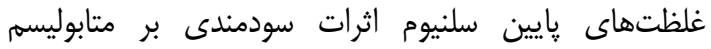

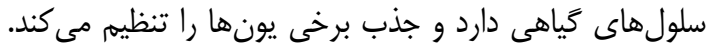

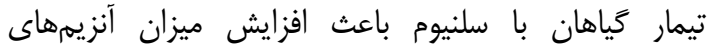

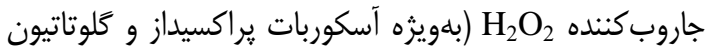

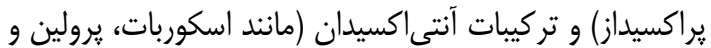

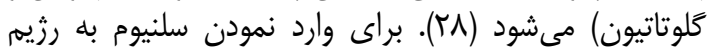

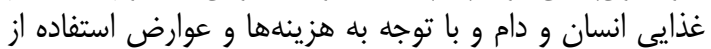

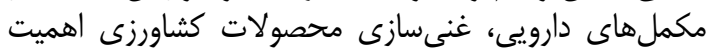

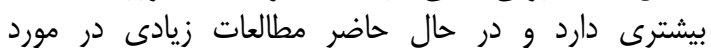

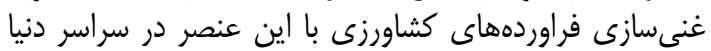

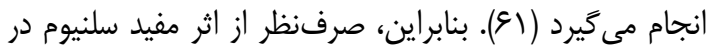

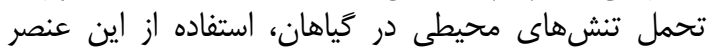

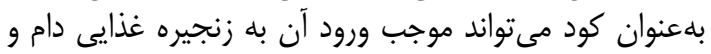

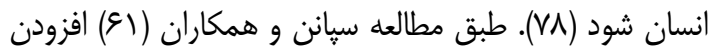

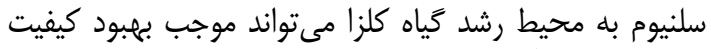

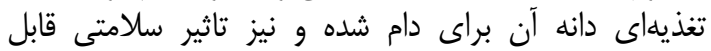

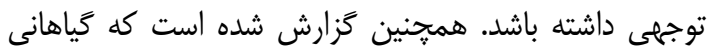

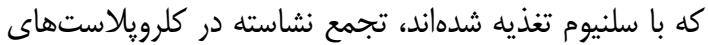

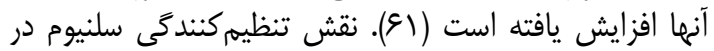

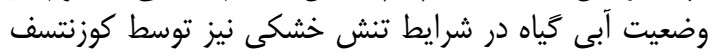

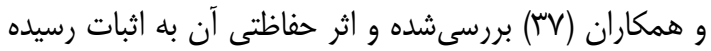

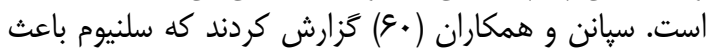

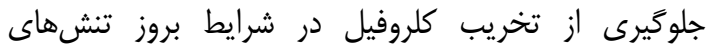

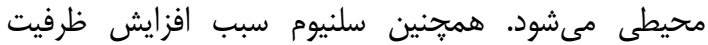

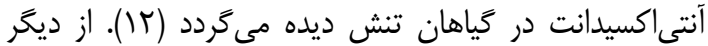

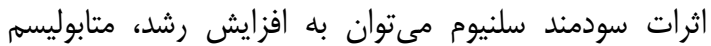

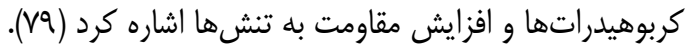

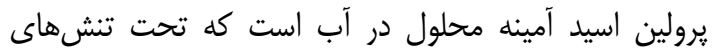

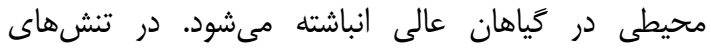

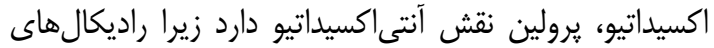

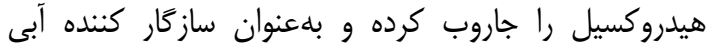

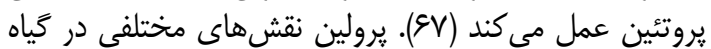

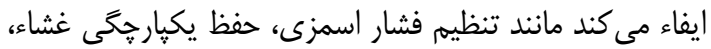

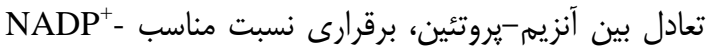
NADPH

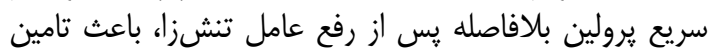

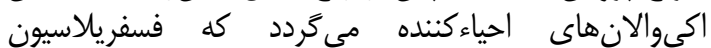

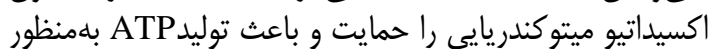

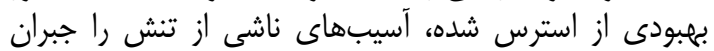

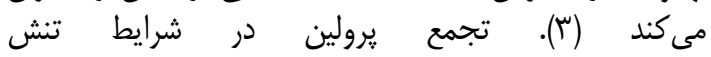




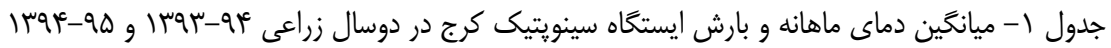
Table 1. Average monthly temperature and precipitation of Karaj synoptic station during two crop years 2014-2015 and 2015-2016

\begin{tabular}{|c|c|c|c|c|c|c|c|c|c|c|}
\hline \multicolumn{2}{|c|}{ بهمن } & \multicolumn{2}{|c|}{ دى } & \multicolumn{2}{|c|}{ آذر } & \multicolumn{2}{|c|}{ آبان } & \multicolumn{2}{|c|}{ مهر } & ماه \\
\hline 149$)^{9}$ & Trar & $11 \% q 4$ & (rar & 1794 & 1rar & $14 q 4$ & (149 & 11494 & (ra & سال \\
\hline$\Lambda / V$ & $F V / \Lambda$ & $10 / 9$ & 9 & TN/G & TI/द & $V V / f$ & $1 \% / V$ & $\Gamma / \Delta$ & $1 \% / f$ & بارش (mm) \\
\hline $4 / 9$ & $V / r$ & $\Delta / 1$ & $\Delta / r$ & 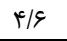 & $8 / \pi$ & $1 \cdot 10$ & $M / T$ & $19 / 5^{c}$ & $|N /|$ & ميانگَين دما (C) \\
\hline \multicolumn{2}{|c|}{ تير } & \multicolumn{2}{|c|}{ خرداد } & \multicolumn{2}{|c|}{ ارديبهشت } & \multicolumn{2}{|c|}{ فروردين } & \multicolumn{2}{|c|}{ اسفند } & ماه \\
\hline $11 \% 9$ & 1199 & $11 \% 9$ & 1raf & 1199 & $1 r q 4$ & $1 \pi 90$ & $11 \% 94$ & 1199 & Iram & سال \\
\hline • & $\cdot$ & - & $9 / 9$ & $\pi$ & $T / T$ & $V D / \Delta$ & $F \Delta / \kappa^{\prime}$ & $\mathrm{IV} / \mathrm{A}$ & $r / r$ & بارش (mm) \\
\hline
\end{tabular}

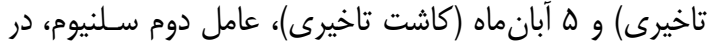

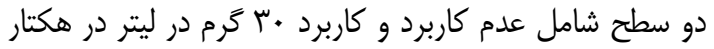

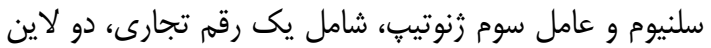

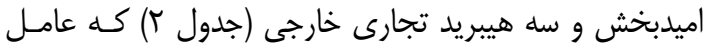

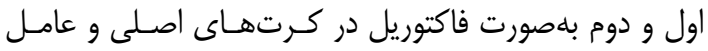

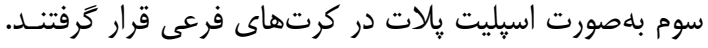

\section{مشخصات آزمايش إز}

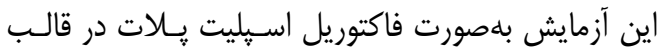

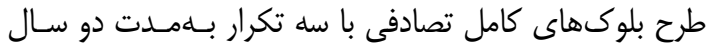

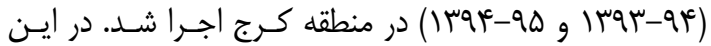

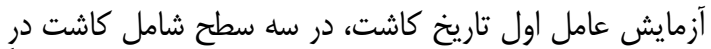

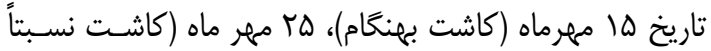

Table 2. Characteristics of the genotypes used

جدول r- مشخصات زنوتيبهاى مورد آزمايش

\begin{tabular}{|c|c|c|c|c|}
\hline منشاء & نوع & تيِ رشد & رنوتيب & رديف \\
\hline سوئد & رقم تجارى & بينابين & Opera & 1 \\
\hline ايران & لاين اميد بخش & زمستانه & L72 & r \\
\hline ايران & لاين اميد بخش & زمستانه & KR1 & r \\
\hline مجارستان & هيبريد & زمستانه & GKH3705 & f \\
\hline مجارستان & هيبريد & زمستانه & GKH0224 & $\Delta$ \\
\hline فرانسه & هيبريد & زمستانه & Neptune & 9 \\
\hline
\end{tabular}

اين صورت كه بذور دو طرف هر رشته و با فاصله از يكديگر و

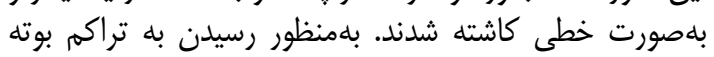

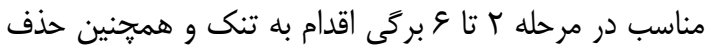

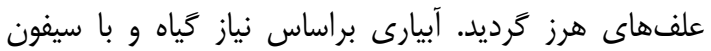

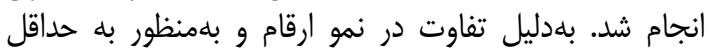

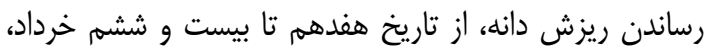

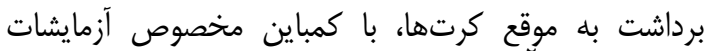
(وينتر اشتايخر) 'انجام شد.

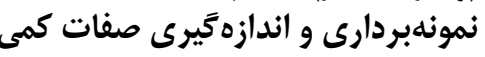

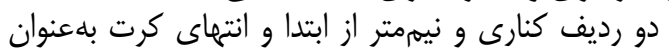

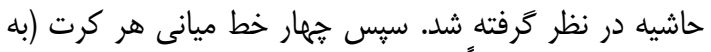

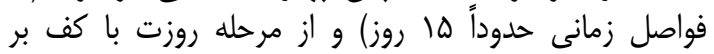

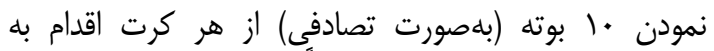

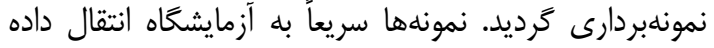

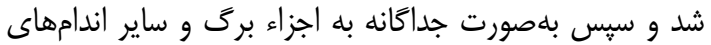
گياه تفكيك گرديد. براى بهدست آوردن قطر طوقه از كوليس استفاده كرديد.

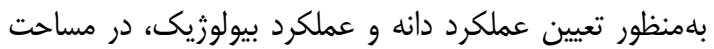

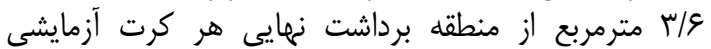

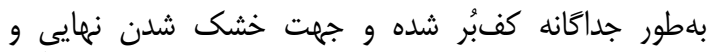

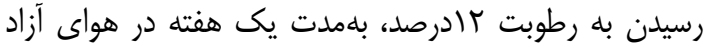
قرار داده شدند.

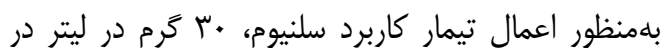

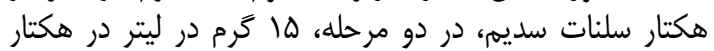

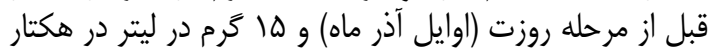

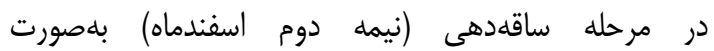

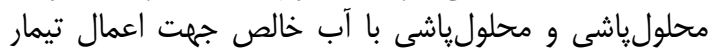
عدم كاربرد سلنيوم، انجام يذيرفت واني

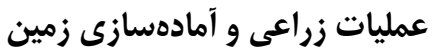

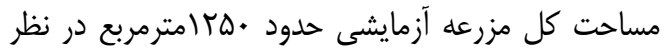

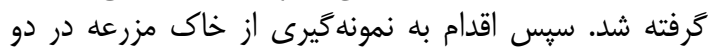

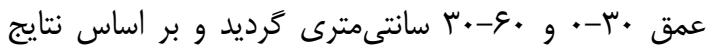

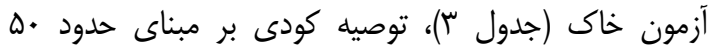

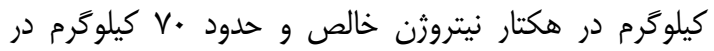

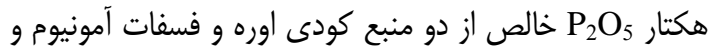

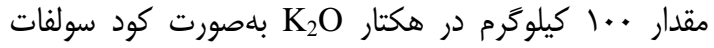

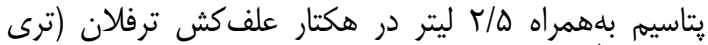

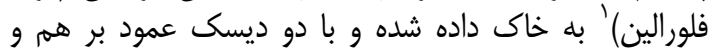
سبك، با خاك مخلوط گرديد.

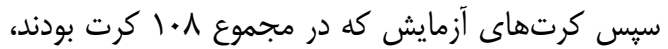

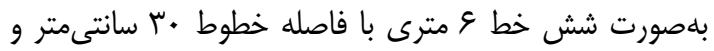

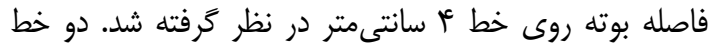

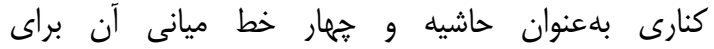
نمونهبردارى و بررسى صفات صنات مختلف، مورد استفاده قرار

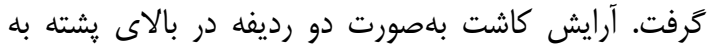


Table 3. Physical and chemical properties of the soil of experimental farm

جدول ب- مشخصات فيزيكى و شيميايى خاك مزرعه آزمايشى

\begin{tabular}{|c|c|c|c|c|c|c|c|c|}
\hline اشباع (\%و مل & $\begin{array}{l}\text { الكتريكى } \\
\text { (ds/m) }\end{array}$ & $\begin{array}{c}\text { خاكنش } \\
\text { خاك } \\
\text { خأ }\end{array}$ & كربن آلى & 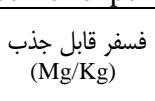 & 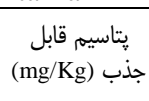 & نيتروث) كل & بافت خاك & عمق نمونهبردارى \\
\hline קq & $1 / \& \Delta$ & $V / q$ &.$/ 91$ & $1 \% / V$ & 198 & .1 .9 & رسى لومى & - r. \\
\hline r & $1 / T F^{C}$ & $V / r$ &.$/ 99$ & $10 / \Lambda$ & $10 \Delta$ & $\cdot / \cdot V$ & رسى لومى & r.-q. \\
\hline
\end{tabular}

مفروضات تجزيه واريانس، بلمنظور آزمون همحنى واريانس

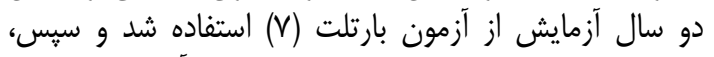
تجزيه واريانس مركب دادهها بر اساس مدل آناست آمارى فاكتوريل

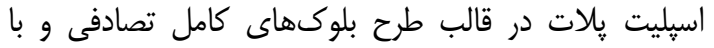

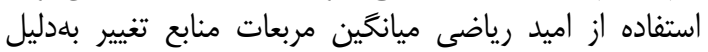

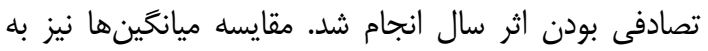

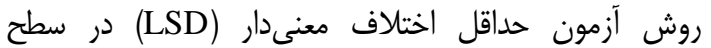

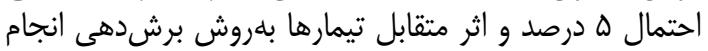

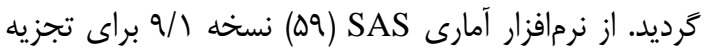
آمارى استفاده شد.

\section{نتايج و بحث زمبث

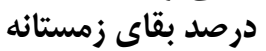

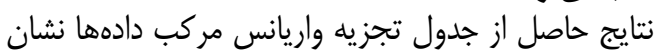

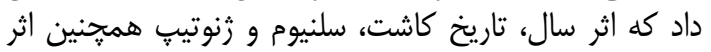

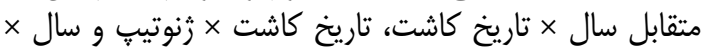

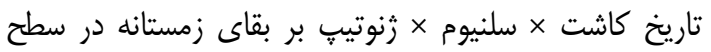

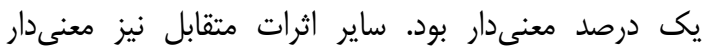

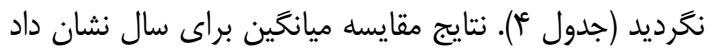

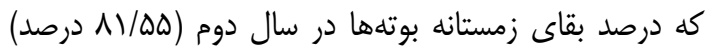

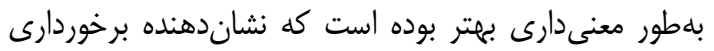

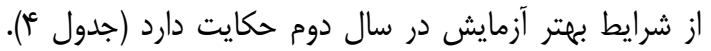

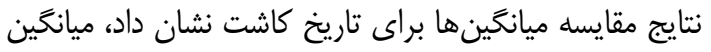

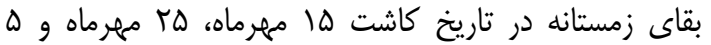

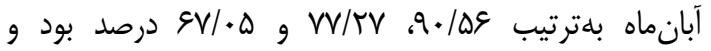

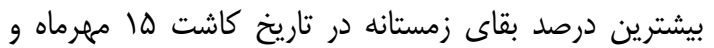

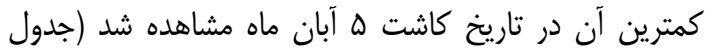

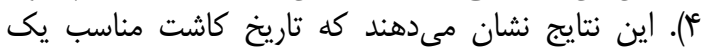

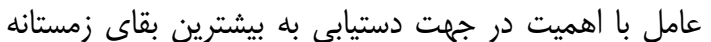

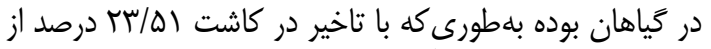

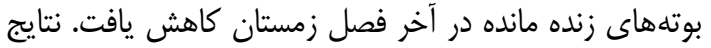

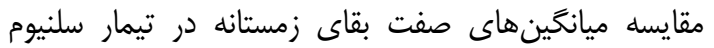

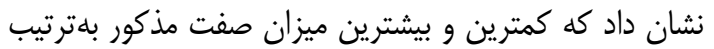

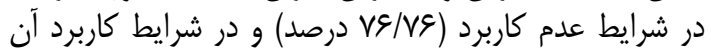

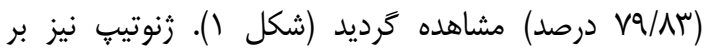

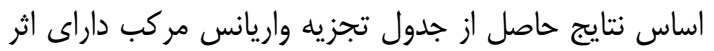

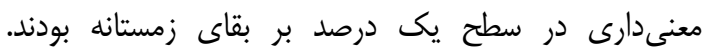

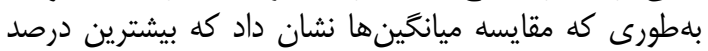

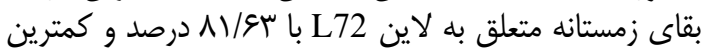

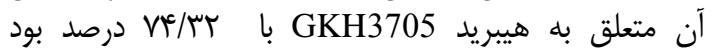

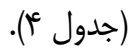

قبل از جداكردن دانه از خورجين، وزن كل بوتهها (برى،

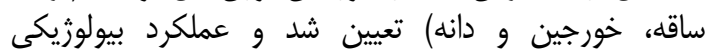

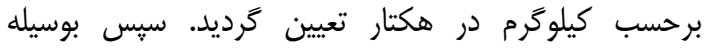

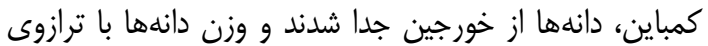

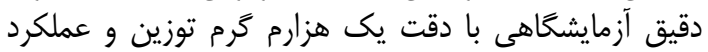
دانه بر حسب كيلوكرم در هكتار محاسبه كر دريد.

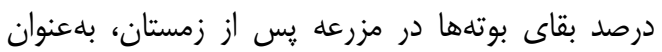

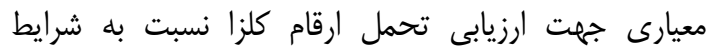

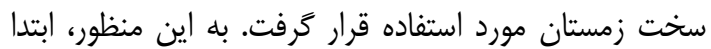

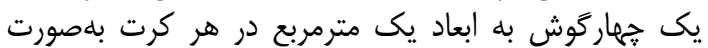

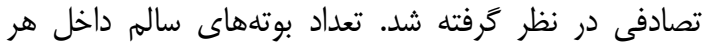

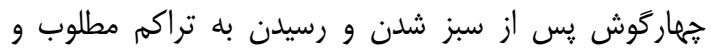

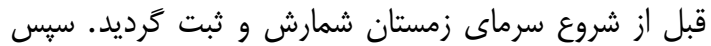

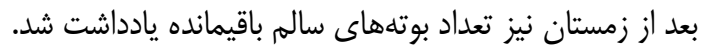

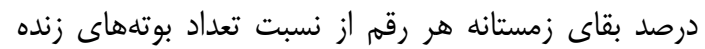
يس از زمستان به تعداد بوتههاى قبل از زمستان محاسبه تداد

شد.

$$
\text { تعديين محتواى نسيى صفات كيفى آب برى }
$$

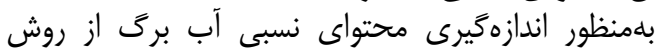

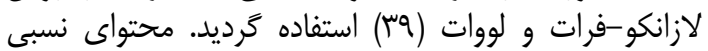
آب بركى، از رابطه زير محاسبه شد:

$R W C(\%)=\left[\frac{(F W-D W)}{(T W-D W)}\right] \times 100$

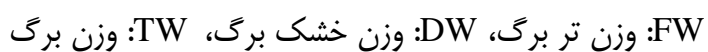

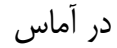

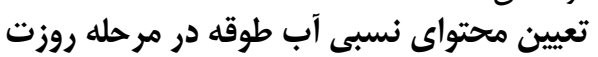

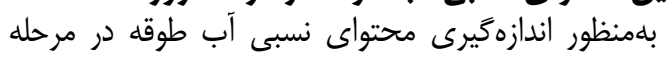

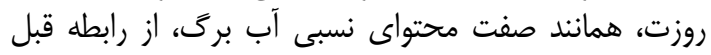
محاسبه شد.

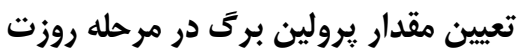

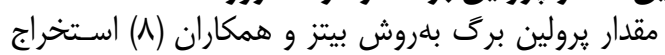

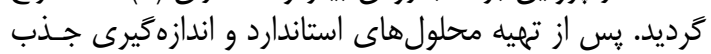

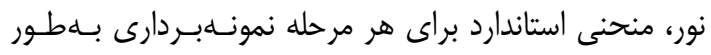

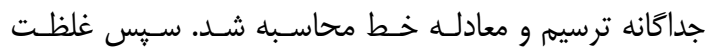

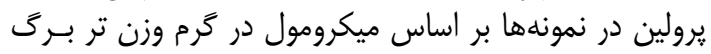
محاسبه گرديد.

\section{تجزيه و تحليل آمارى دادهها}

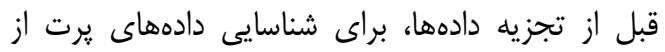

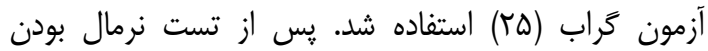

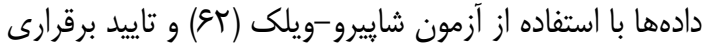




\section{عملكرد دانه}

نتايج حاصل از تجزيه واريانس مركب دادهانها نارئ نشان داد

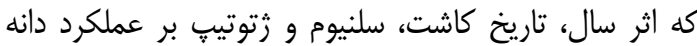

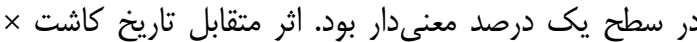

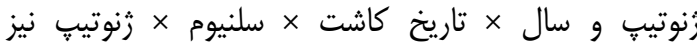

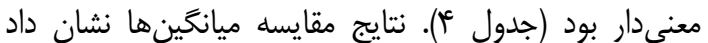

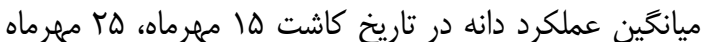

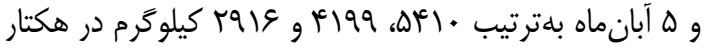

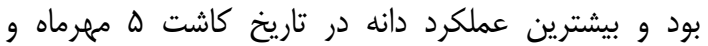

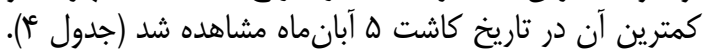

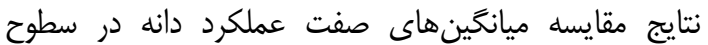

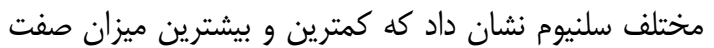

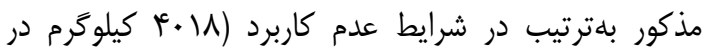

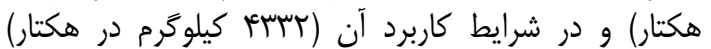

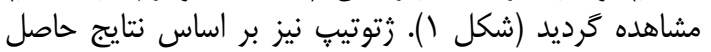

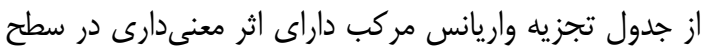

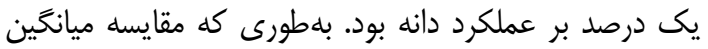

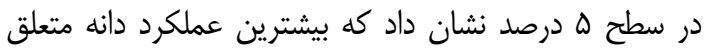

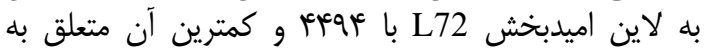

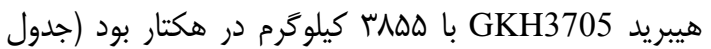
()

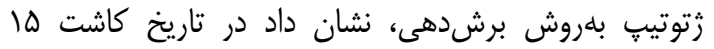

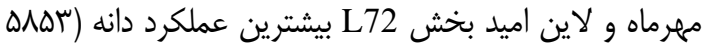

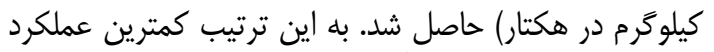

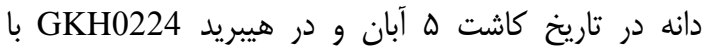

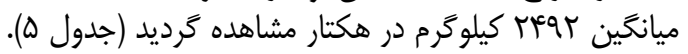

عملكرد بيولوزيكى

نتايج حاصل از تجزيه واريانس مركب تارئ دادئ دادها نشان داد

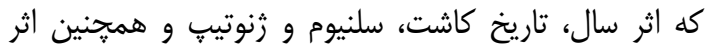

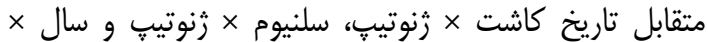

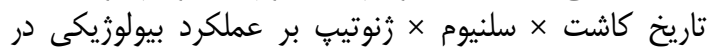

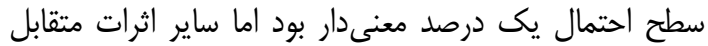

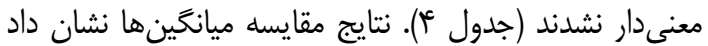

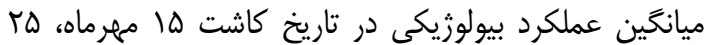

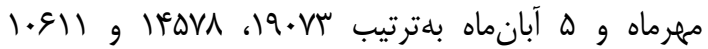
كيلوگرم در هكتار بود و بيشترين عملكرد بان بيولوزيكى در دان تاريخ

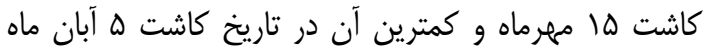

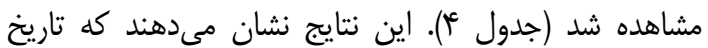

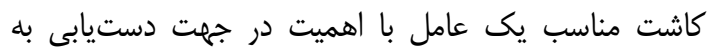

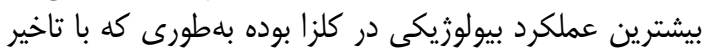

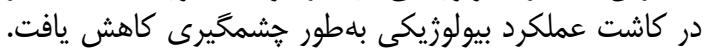

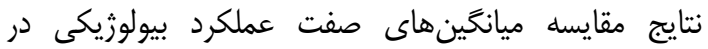

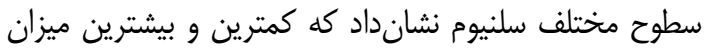

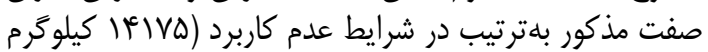

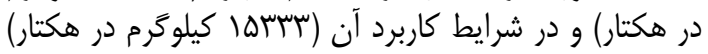

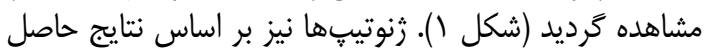

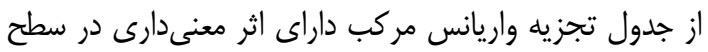

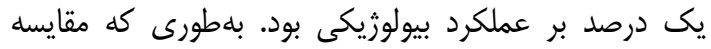

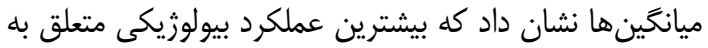

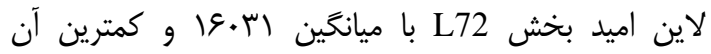

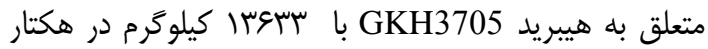
بود (جدول مأ).

جدول عا- تجزيه واريانس و مقايسه ميانخين اثرات اصلى در ارقام و لاينهاى كلزا Table 4. Analysis of variance and comparison of means of main effects on rapeseed cultivars and lines

\begin{tabular}{|c|c|c|c|c|c|c|c|c|c|}
\hline 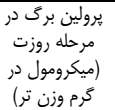 & نسبى آب & 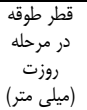 & محر محتواى نسبى روقه در & شراخت & 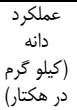 & 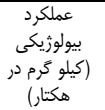 & زمستاى & & آزادى \\
\hline ** & $\mathrm{ns}$ & $\mathrm{ns}$ & ** & $\mathrm{ns}$ & ** & ** & ** & & \\
\hline $\mid V / q q^{a}$ & $9.1 .9^{\mathrm{a}}$ & $1 . / r 1^{\mathrm{a}}$ & $99 / \sim^{\circ} \Lambda^{\circ}$ & $r V / \Lambda \Delta^{a}$ & $r \wedge 11^{0}$ & $\mid r V I V^{0}$ & $V Q / \cdot \varphi^{\mathrm{D}}$ & سال اول & سال \\
\hline $1 T / F F^{D}$ & $19 / \mathrm{n}^{\mathrm{a}}$ & $1 . / r \Delta^{\mathrm{a}}$ & $v \cdot / N^{a}$ & $r N / V^{a}$ & $r \Delta q^{a}$ & lovala & $\Lambda \mathrm{N} / \Delta \Delta^{\mathrm{a}}$ & سال دوم & \\
\hline *** & ** & *** & *** & $\mathrm{ns}$ & *** & $* *$ & $* *$ & & تاريخ كاشت \\
\hline $11 / \pi \mathrm{F}^{\mathrm{c}}$ & $৭ \Upsilon / ৭ q^{a}$ & $w / v \bullet^{a}$ & $99 / \% \cdot \cdot^{\mathrm{c}}$ & TN/Fra & $\Delta F 1 \cdot{ }^{a}$ & $19 \cdot v r^{\mathrm{a}}$ & $9 \cdot / \Delta \gamma^{\mathrm{a}}$ & ها مهرماه & \\
\hline $\mid \Delta / \Delta \Delta^{D}$ & $\Lambda 9 / \Delta \Gamma^{D}$ & $1 . / \% \cdot{ }^{D}$ & $99 / r^{\circ}$ & TN/AF $\mathrm{a}^{\mathrm{a}}$ & $1199^{\circ}$ & $1 F \Delta V \Lambda^{\circ}$ & $W / T V^{D}$ & SA Tهرماه & r \\
\hline$r \cdot / r)^{\mathrm{a}}$ & $\Lambda V / / N^{c}$ & $9 / 90^{\circ}$ & $v^{e} / \mu^{a}$ & $T V / \Delta T^{\mathrm{a}}$ & rq१ $\varepsilon^{\mathrm{c}}$ & $1.911^{\mathrm{c}}$ & $9 \mathrm{~V} / \cdot 0^{\mathrm{D}}$ & هـ آبان ماه & \\
\hline ** & $*$ & *** & ** & $\mathrm{ns}$ & *** & *** & $* *$ & & 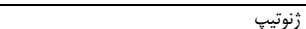 \\
\hline $10 / \cdot 1^{0}$ & 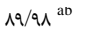 & $1 . / 90^{\circ}$ & $89 / 44^{\mathrm{bc}}$ & $\mu r / r^{a}$ & Frrta & $10 \cdot V V^{\circ}$ & $v a / T v^{a}$ & Opera & \\
\hline$|F / F|^{D}$ & $9 \cdot / \mathrm{v}^{\mathrm{a}}$ & $11 / 1 f^{a}$ & $9 q / 19^{c}$ & $\kappa r / \& q^{a}$ & $r q q q^{a}$ & $|9 \cdot|^{\mathrm{a}}$ & $\Lambda \mathrm{s} / \mathrm{q}^{\mathrm{a}}$ & L72 & \\
\hline $19 / 0 \cdot^{a}$ & $\Lambda 9 / \% \varepsilon^{D}$ & $q / \Delta \mathcal{F}^{\mathrm{c}}$ & $v \cdot / v \cdot{ }^{a b}$ & $+1 / \mathbb{S}^{\circ}$ & $r Q \Delta f^{\circ}$ & $\mid$ |rq..$^{\mathrm{c}}$ & $V Q / q \varphi^{D}$ & KR1 & $\Delta$ \\
\hline$|\varepsilon / \Delta|^{a}$ & $\Lambda 9 / \propto \psi^{D}$ & $\checkmark / 94^{c}$ & $v \cdot / \Lambda r^{\mathrm{ab}}$ & $\sim / \Lambda \Delta^{D}$ & $r q \Delta \xi^{D}$ & $14 \cdot r^{\mathrm{c}}$ & $v \xi / T^{D}$ & GKH3705 & \\
\hline$|\varepsilon / \wedge|^{a}$ & $19 / \pi r^{D}$ & $q / r \Lambda^{c}$ & $V / r \cdot{ }^{a}$ & $F / / k^{\circ}$ & $r \Lambda \Delta \Delta^{\circ}$ & ( & $V \in / \pi r^{D}$ & GKH0224 & \\
\hline$\| F / N r^{D}$ & $9 . / 81^{a}$ & $11 / \cdot e^{a}$ & $s q / r q^{D C}$ & $\mu r / \psi^{* a+}$ & $\operatorname{res}^{a}$ & $1 \Delta \wedge r \xi^{a}$ & $\Lambda \mathrm{\Lambda} / \mathrm{\sigma}^{\mathrm{a}}$ & Neptune & \\
\hline$* *$ & ns & $*$ & ns & ns & $\mathrm{ns}$ & $\mathrm{ns}$ & $* *$ & $\frac{T}{r}$ & سال × تاريخ كاشت \\
\hline$*$ & $*$ & $* *$ & $*$ & ns & $* *$ & $* *$ & $* *$ & 1 & سلنيوم \\
\hline ns & ns & ns & ns & ns & ns & ns & ns & 1 & سال × سلنيوم \\
\hline $\mathrm{ns}$ & ns & ns & $\mathrm{ns}$ & ns & ns & ns & $\mathrm{ns}$ & r & تاريخ كاشت × سلنيوم \\
\hline * & ns & ns & ns & ns & ns & ns & ns & r & سال × تاريخ كاشت × سلنيوم \\
\hline ns & ns & ns & ns & ns & ns & ns & $\mathrm{ns}$ & $\Delta$ & سال × • رنوتيب \\
\hline ** & ns & $* *$ & ns & ns & $* *$ & ** & $* *$ & 1. & تاريخ كاشت × زثوتيب \\
\hline ns & ns & ns & ns & ns & ns & ** & $\mathrm{ns}$ & $\Delta$ & سلنيوم × رنوتيب \\
\hline * & ns & ns & ns & ns & ns & ns & $\mathrm{ns}$ & 1. & سال × تاريخ كاشت × رثنوتيب \\
\hline ns & ns & ns & ns & ns & ns & ns & ns & $\Delta$ & سال × سلنيوم × زنوتيب \\
\hline ns & ns & ns & ns & ns & ns & $\mathrm{ns}$ & $\mathrm{ns}$ & 1. & تاريخ كاشت × سلنيوم × رثنوتيب \\
\hline$* *$ & ns & ns & $* *$ & ns & $* *$ & $* *$ & $* *$ & 1. & سال × تاريخ كاشت × سلنيوم × رنوتيت \\
\hline$\Delta / 9 \Lambda$ & $r / \mp \Lambda$ & $N / 4 \mathrm{~V}$ & $F / \Lambda$ & $\mid F / \pi f$ & $11 / v 9$ & S/TV & $8 / 91$ & & ضريب تغييرات (درصد) \\
\hline
\end{tabular}



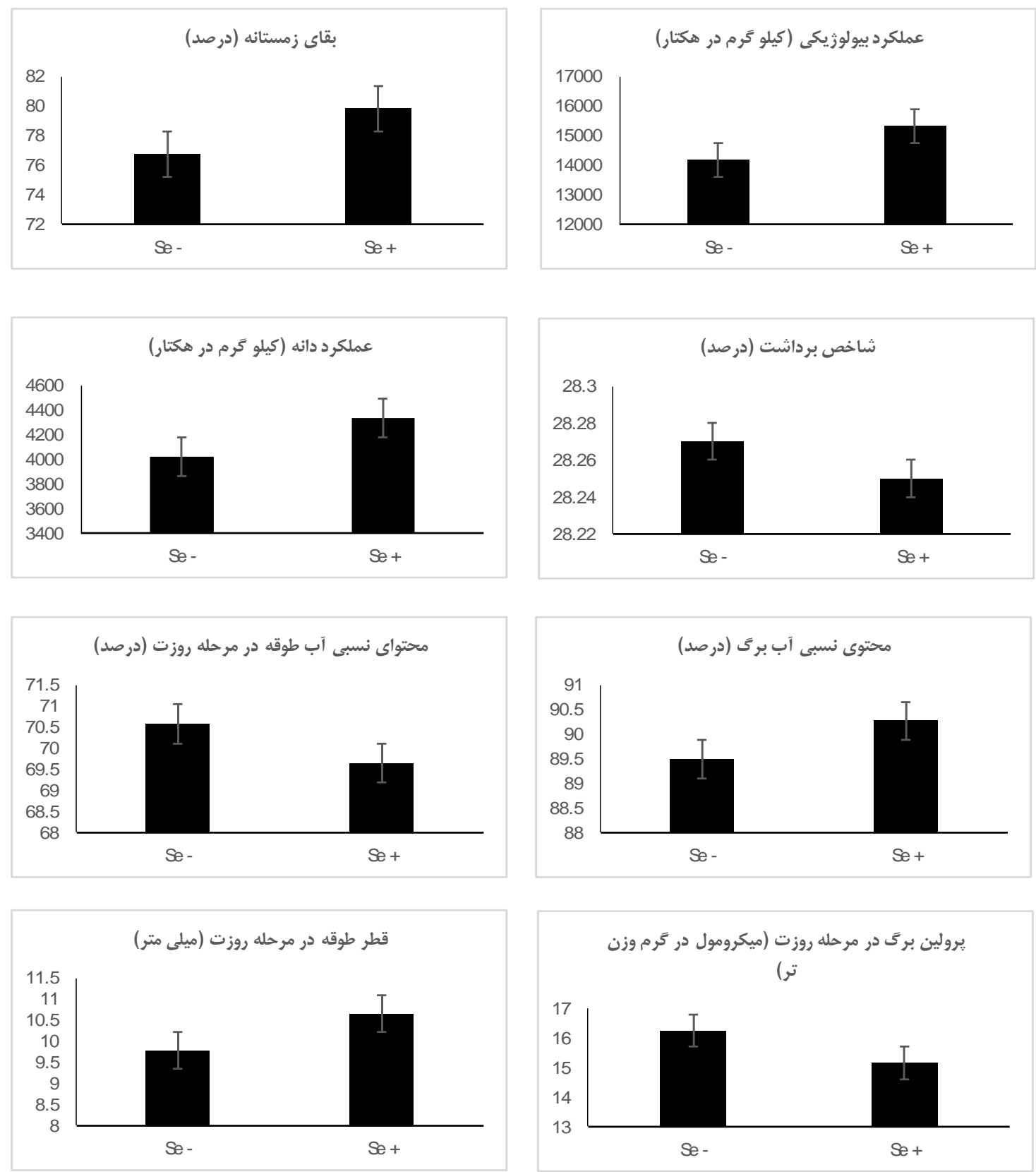

شكل ا- تاثير عدم كاربرد (Se) و كاربرد (Se) محلولياشى سلنيوم بر خصوصيات كمى و كيفى ارقام كلزا در شرايط تنش كَرمايى آخر فصل.

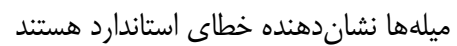

Figure 1. The effect of non-application ( $\left.\mathrm{Se}^{-}\right)$and application $\left(\mathrm{Se}^{+}\right)$of selenium foliar application on quantitative and qualitative characteristics of rapeseed cultivars in late-season heat stress conditions. Bars show standard errors

•

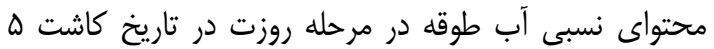

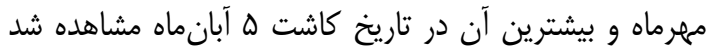

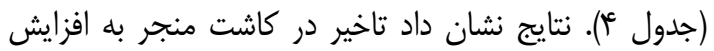

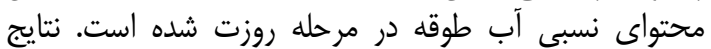

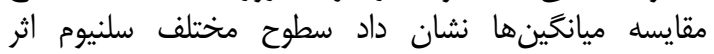

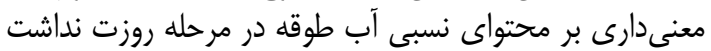

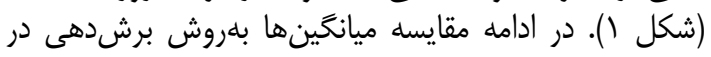

محتواى نسبى آب طوقه در مرحله روزت

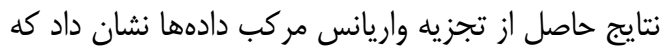

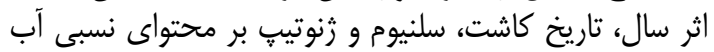

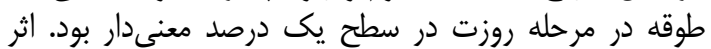

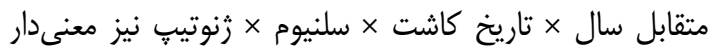

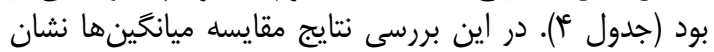

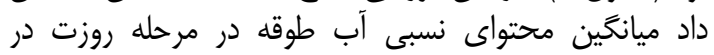

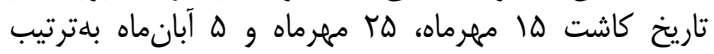


كاشت ها آبانماه مشاهده شد (جدول عأ). نتايج نشان داد تاخير

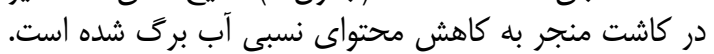

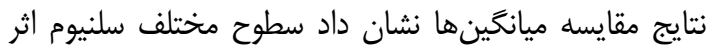

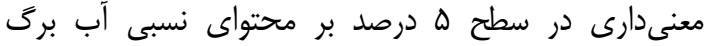

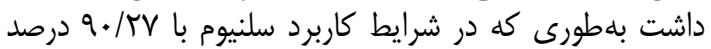

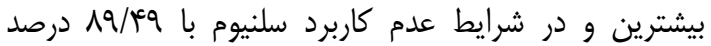

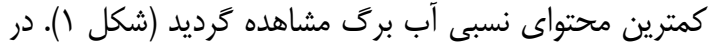

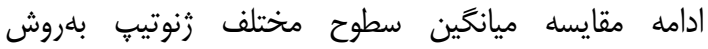

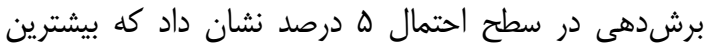

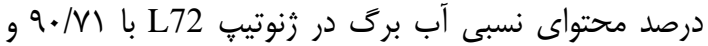

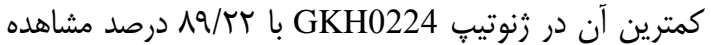

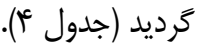

ميزان يرولين برتى در مر حله روزي

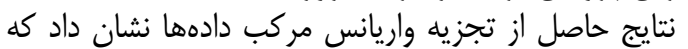

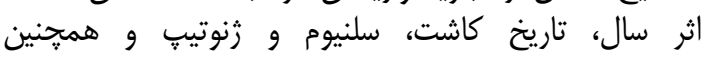

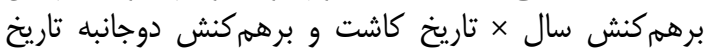

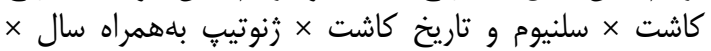

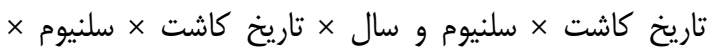

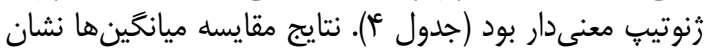

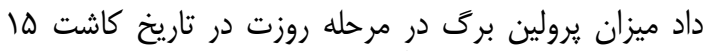

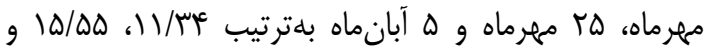

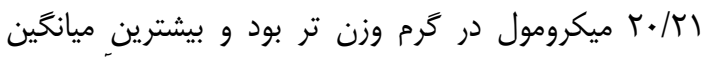

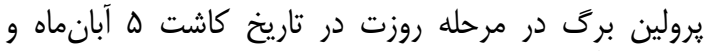

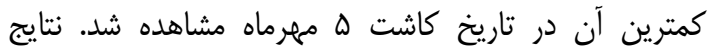

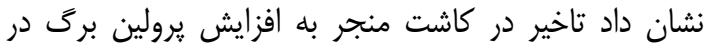

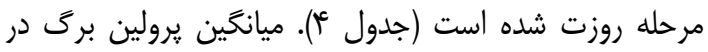

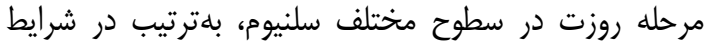

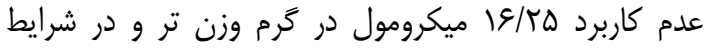

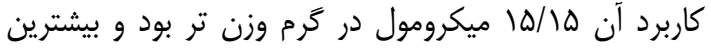

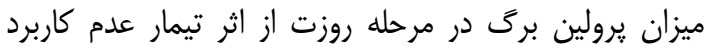

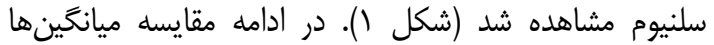

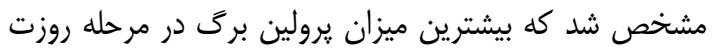

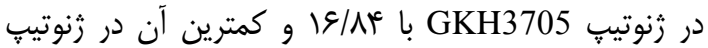

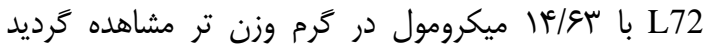

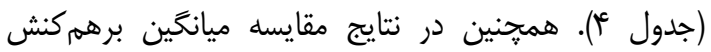

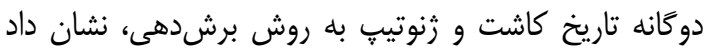

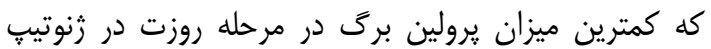

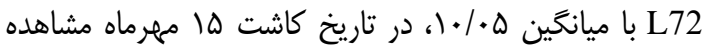

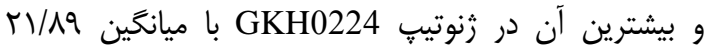
ميكرومول در كرم وزن تر، در تاريخ كاشت ها آبانماه مشاهده

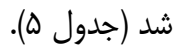

سطح ه درصد نشان داد كه بيشترين ميانگين محتواى نسبى برائ

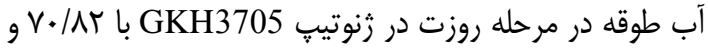

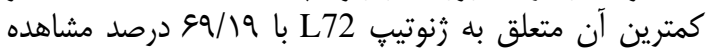

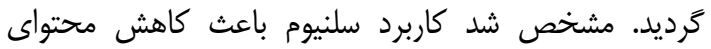

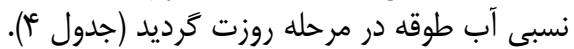

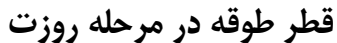

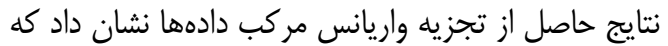

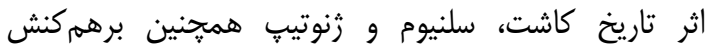

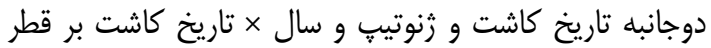

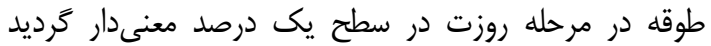

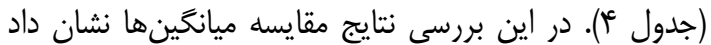

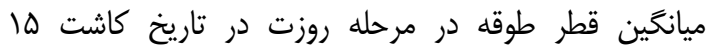

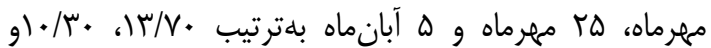

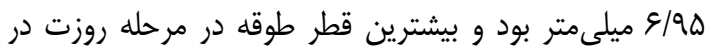

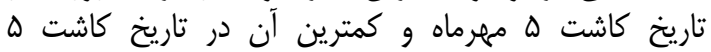

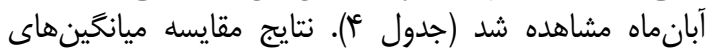

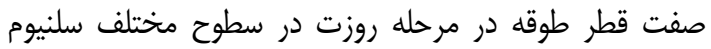

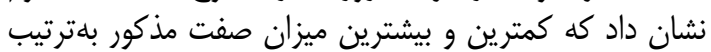

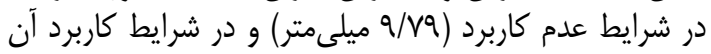

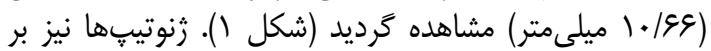

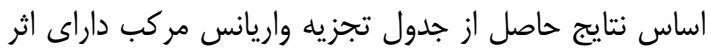

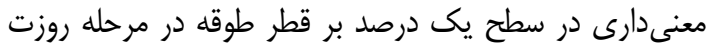

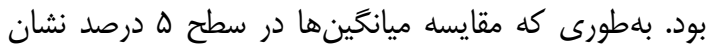

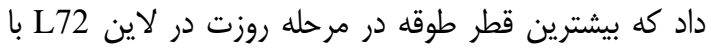

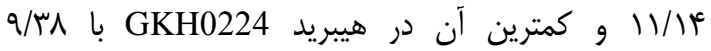

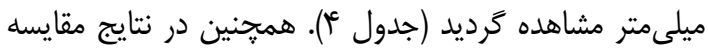

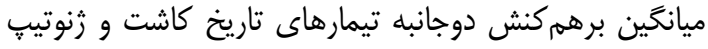

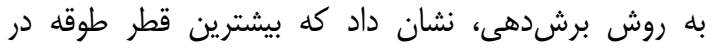

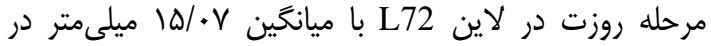

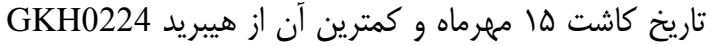
با ميانكين ..19 ميلىمتر در تاريخ كاشت ها ها آبانماه مشاهده شد (جدول ه).

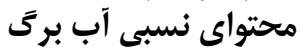

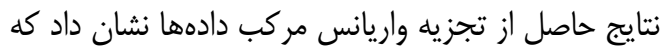

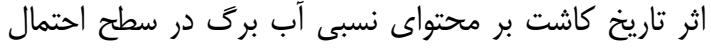

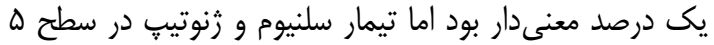

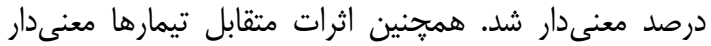

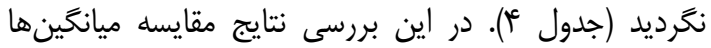

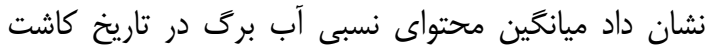

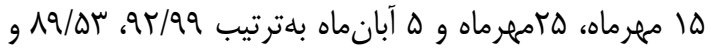

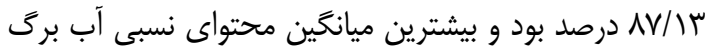

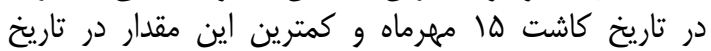


جدول ه- برهمكنش دوجانبه تاريخ كاشت × زنوتيب بر خصوصيات كمى و كيفى ارقام كلزا در شرايط تنش كرمايى آخر فصل Table 5. Interaction of planting datexgenotype on quantitative and qualitative characteristics of rapeseed cultivars under end-season heat stress

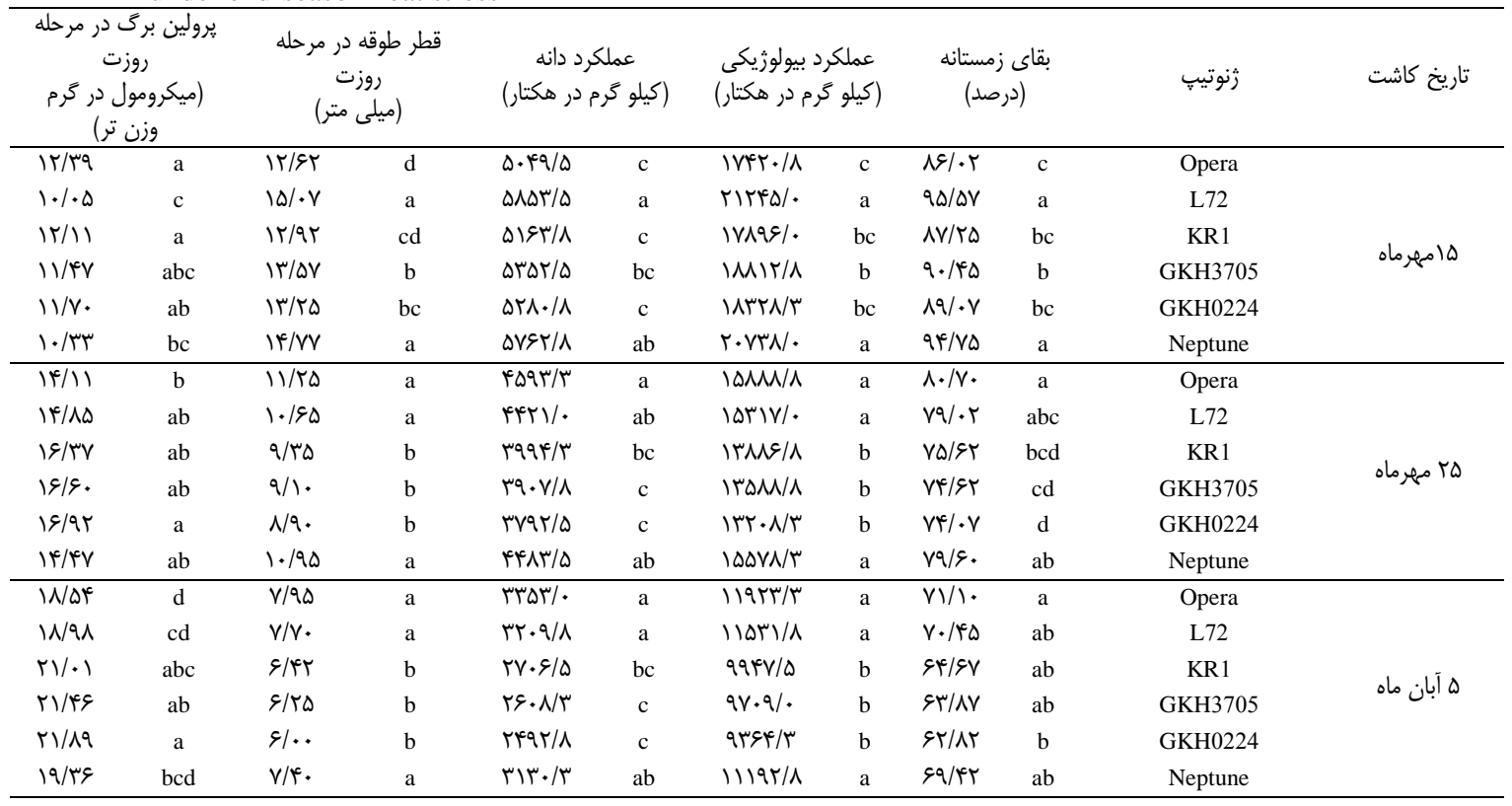

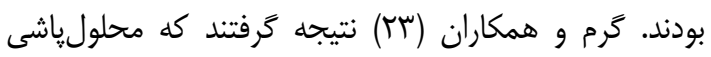

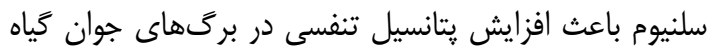

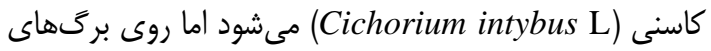

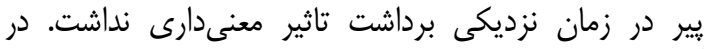

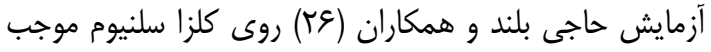

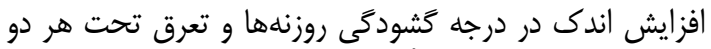

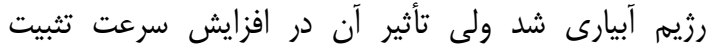

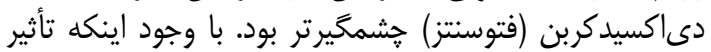

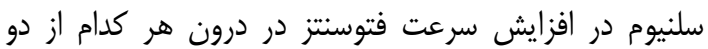

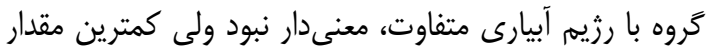

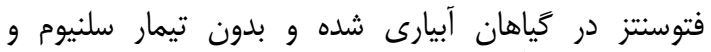
بيشترين مقدار آن در كياهان تحت تيمار همزمان خشكى تشى و سلنيوم مشاهده شد. تاخير در كاشت منجر به كاهش و افت عملكرد در كياهان

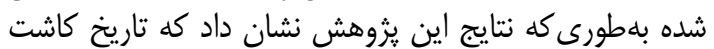

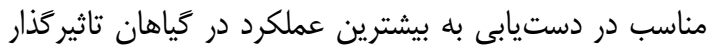

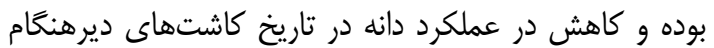

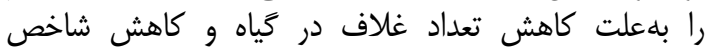

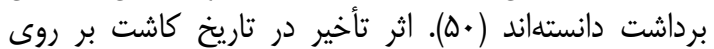

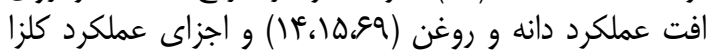

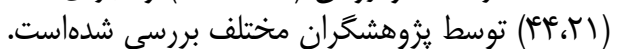

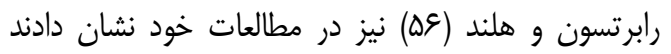

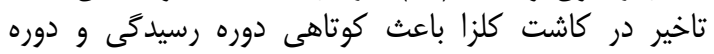

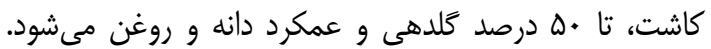

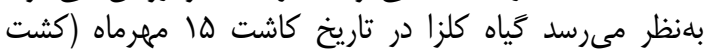

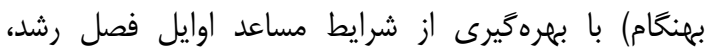

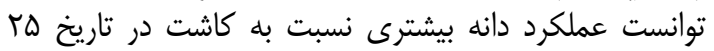
مهر و ه آبانماه (كشت تاخيرى) داشته باشد. نتايج
درصد بقاى زمستانه بوتهها در مزرعه، بلعنوان معيارى جهت ارزيابى تحمل ارقام زمستانه كلزا نسبت بران به شرايط

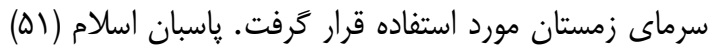

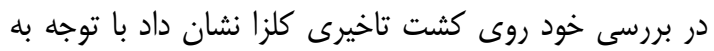

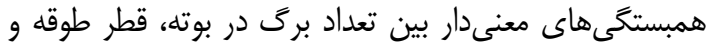

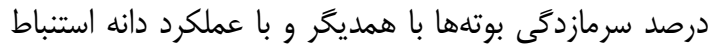

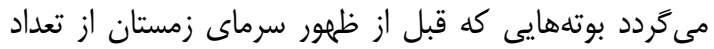

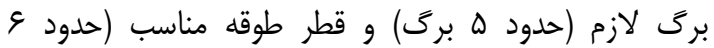

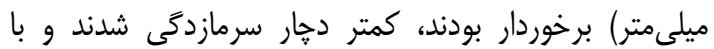

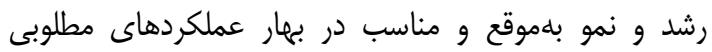

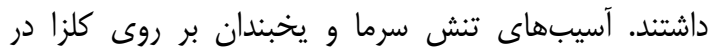

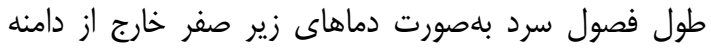

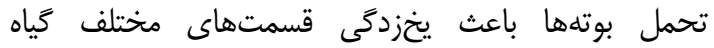

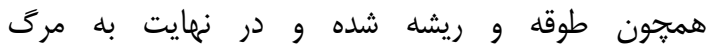

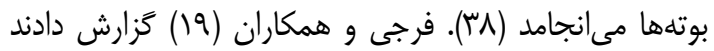

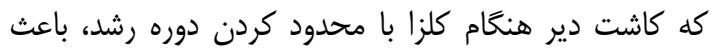

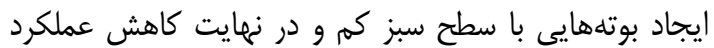

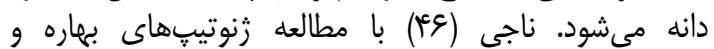

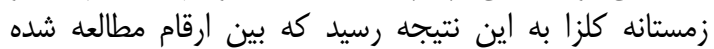

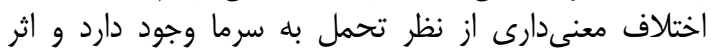

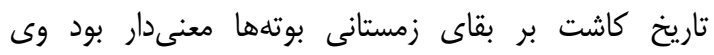

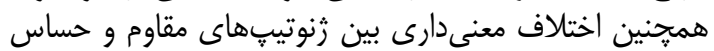

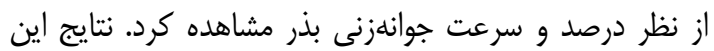
يزوهش نشان داد كه كاربرد سلنيوم توانسته كياهانى بن باني

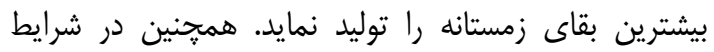

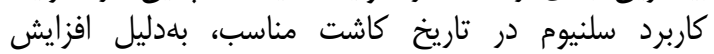

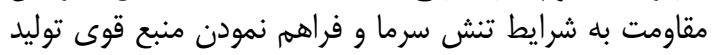
مواد فتوسنتزى، از درصد بقاى زمستانه بيشترى برخوردار 
تيمارهاى كم آبيارى در مراحل كلدهى و خورجيندهى سبب إنب

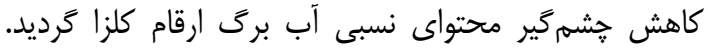

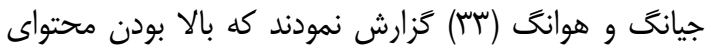

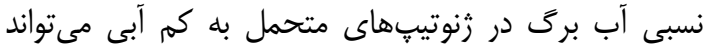

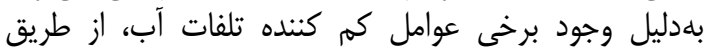

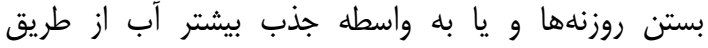

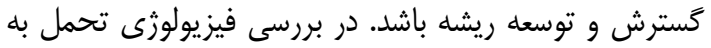

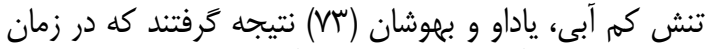

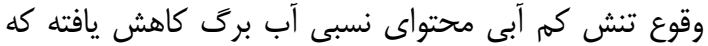

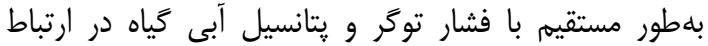

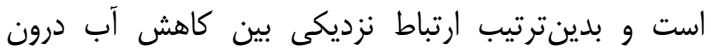

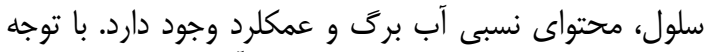

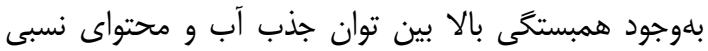

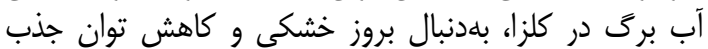

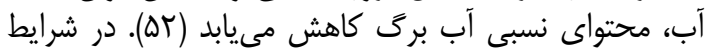

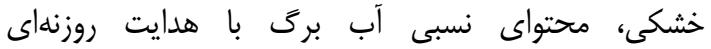

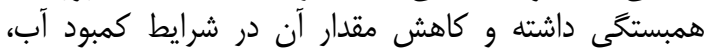

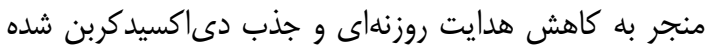

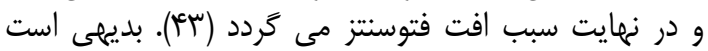

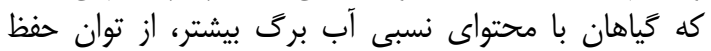

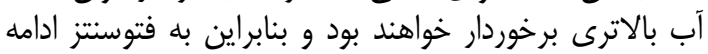

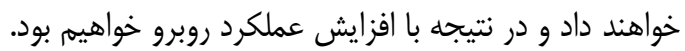

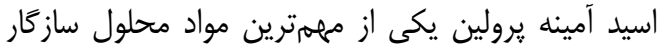

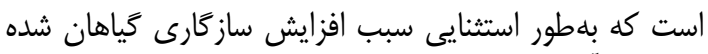

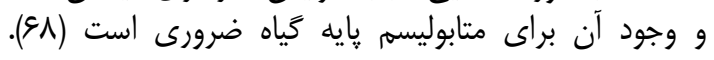

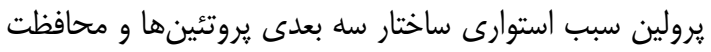

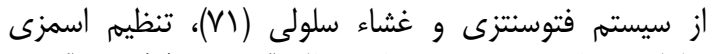

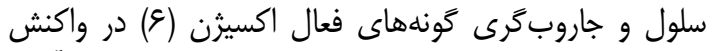

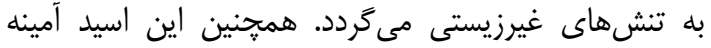

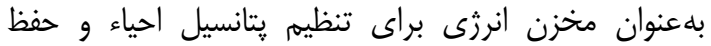

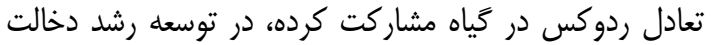

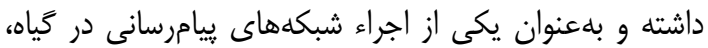

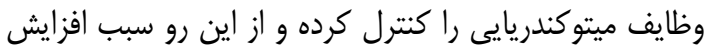

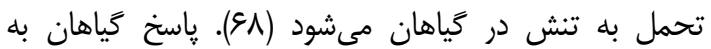

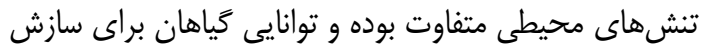

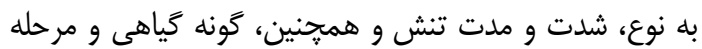

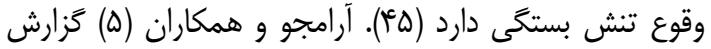

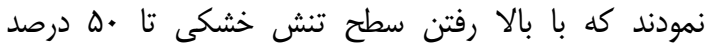

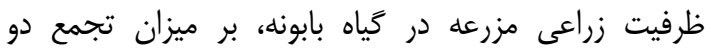

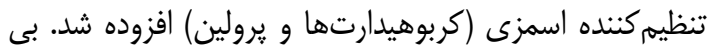

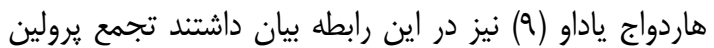

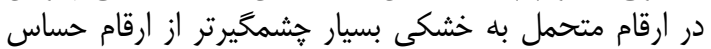

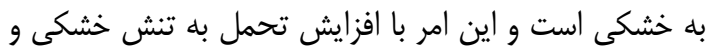

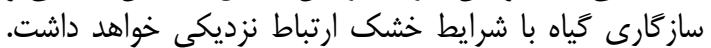

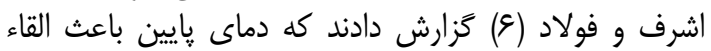

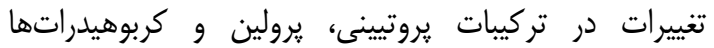

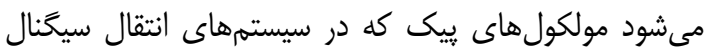

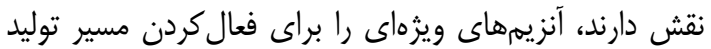
يرولين ايجاد مى كنند تا باعث تشكيل يا تنظيم فعائ فراليت
يزروهشهاى مختلف نشان مىدهد افزايش عملكرد دانه بر اثر برائ

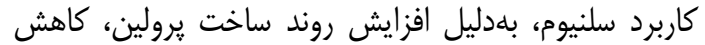

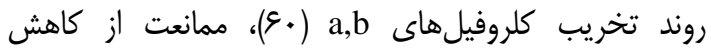

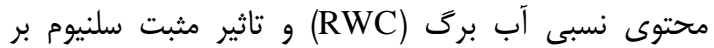
شاخصهاى فيزيولوزيكى رشد (RGR,CGR,LAI,TDW)

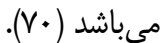
با توجه به اينكه شاخص برداشت معيارى از كارايى انتقال

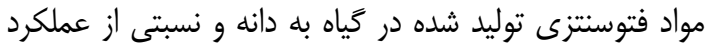

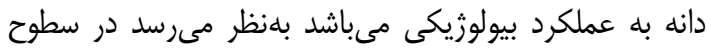

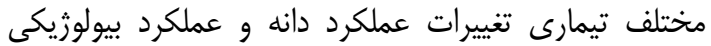

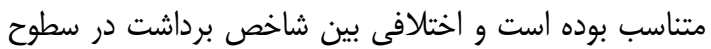

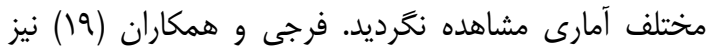

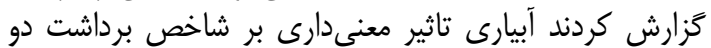
رقم كلزاى بهاره نداشته است.

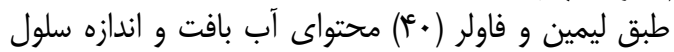

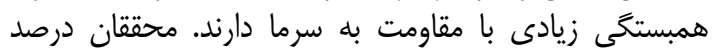

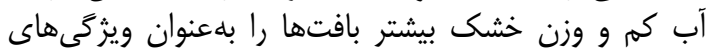

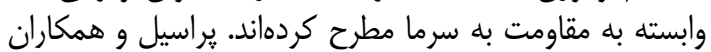
(DF)

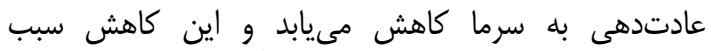

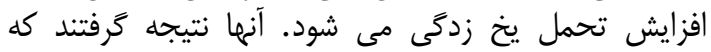

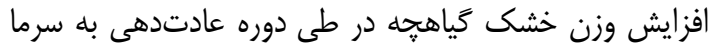

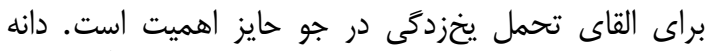

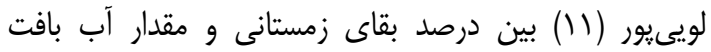

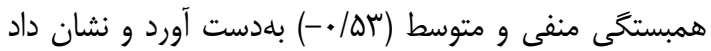

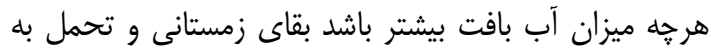

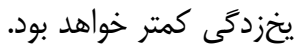

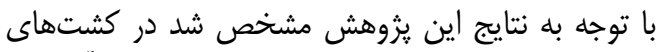

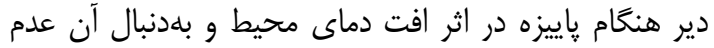

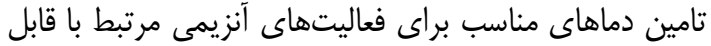

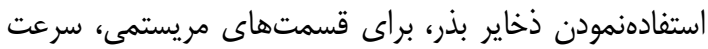

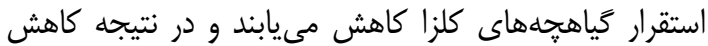

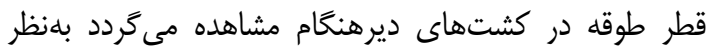

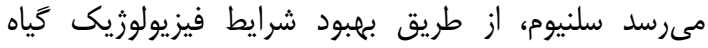
مىتواند موجب بهبود شرايط رشد كياه و حصول قطر طوقي فيز بيشتر شود.

محتواى آبى بركَها بهاعنوان عاملى براى تعيين سطح

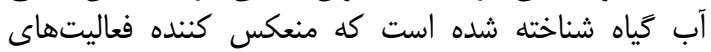

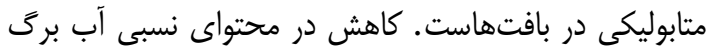

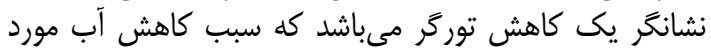

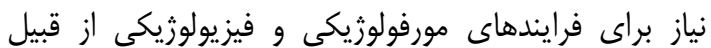

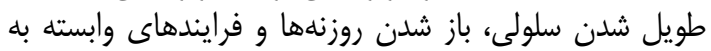

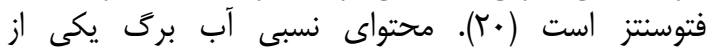

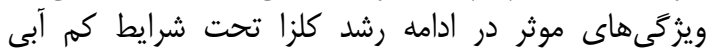

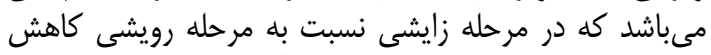

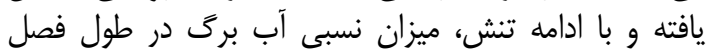

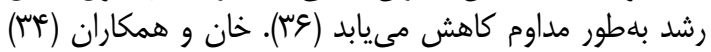

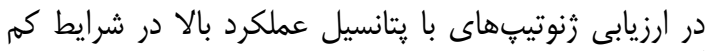

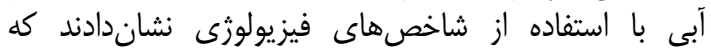




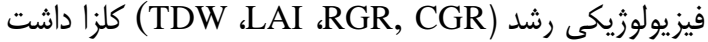
(V•) צ ميلى

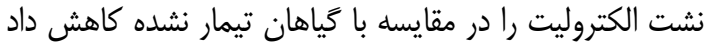

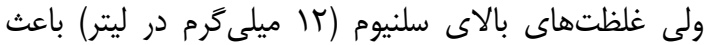
افزايش درصد نشت الكتروليت كرديد.

صفات كمى و كيفى كلزا در اين آزمايش تحت كأنتأثير تاريخ

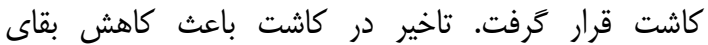

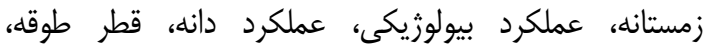

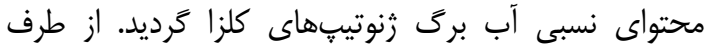

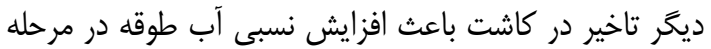

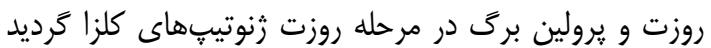

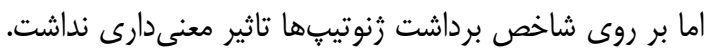

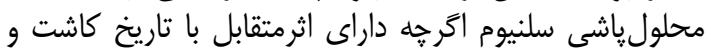

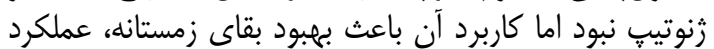

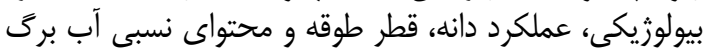

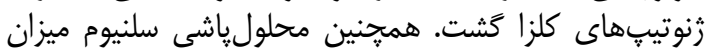

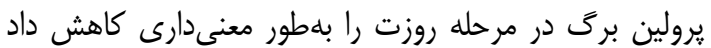

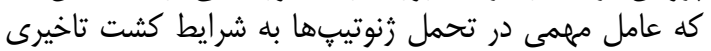

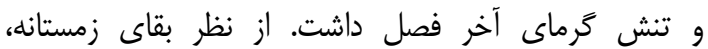

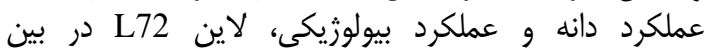

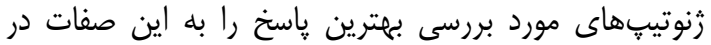

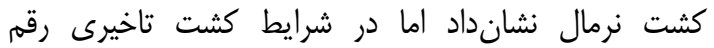

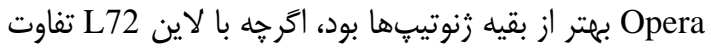
معنى دارى نداشت.

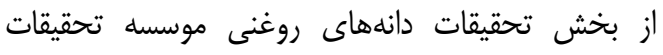

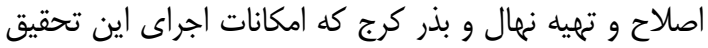

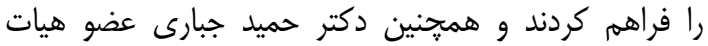

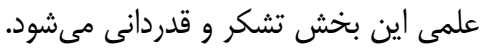

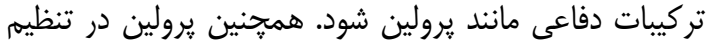

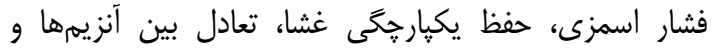

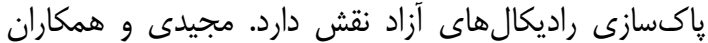

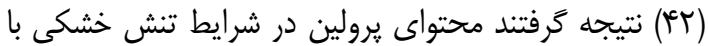

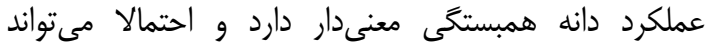

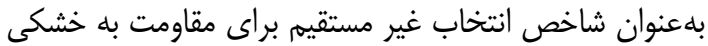

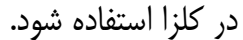

با توجه بلنتايج بررسى اين آزمايش مشاده

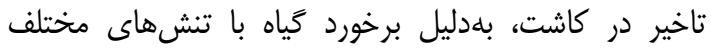

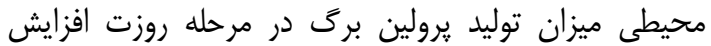

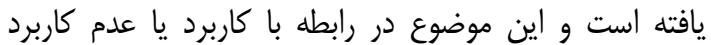

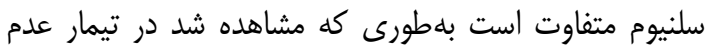

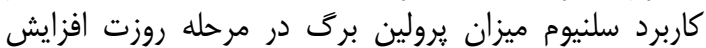

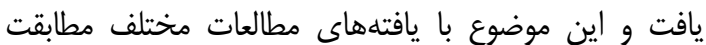
دارد. حاجى بلند و همكاران (عَ) نشان دادند كه اثر سلنيوم در

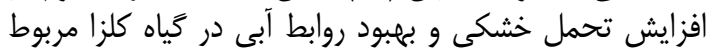

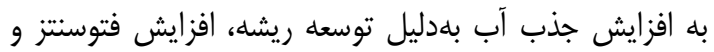

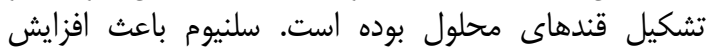

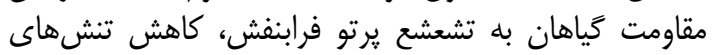

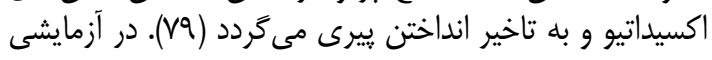

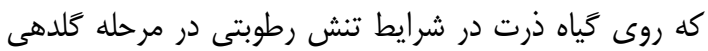

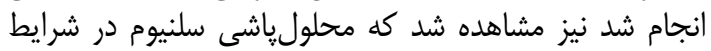

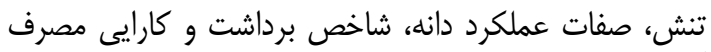

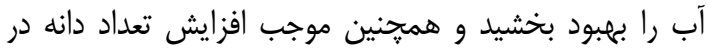

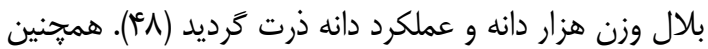

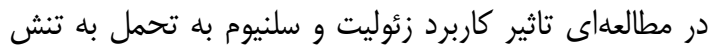

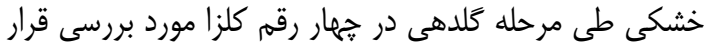

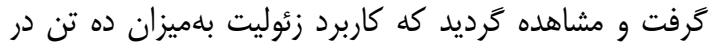

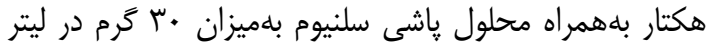

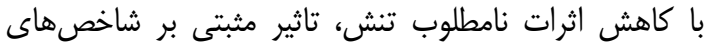

منابع

1. Abbas, S.M. 2012. Effects of low temperature and selenium application on growth and the physiological changes in sorghum seedlings. Journal of Stress Physiology and Biochemistry, 8(1): 268-286.

2. Adamsen, F. and T. Coffelt. 2005. Planting date effects on flowering, seed yield, and oil content of rape and crambe cultivars. Industrial Crops and Products, 21(3): 293-307.

3. Alia Saradhi, P. and P. Mohanty. 1993. Proline in relation to free radical production in seedlings of Brassica juncea raised under sodium chloride stress. Plant and Soil, 155(156): 497-500.

4. Anonymous. 2012. Annual Report. Iranian Vegtable Oil Industry Association.

5. Arazmjo, E., M. Heidari and A. Ghanbari. 2010. Effect of water stress and type of fertilizer on yield and quality of chamomile (Matricaria chamomilla L.). Iranian Journal of Crop Sciences, 12(2): 100111 (In Persian).

6. Ashraf, M. and M. Foolad. 2007. Roles of glycine betaine and proline in improving plant abiotic stress resistance. Environmental and Experimental Botany, 59(2): 206-216.

7. Bartlett, M. 1937. A test for homogeneity of variances. A test for homogeneity of variances. Proceedings of the Royal Society of London, 160: 268-282.

8. Bates, L., R. Waldren and I. Teare. 1973. Rapid determination of free proline for water-stress studies. Plant and Soil, 39(1): 205-207.

9. Bhardwaj, J. and S.K. Yadav. 2012 .Comparative study on biochemical parameters and antioxidant enzymes in a drought tolerant and a sensitive variety of Horsegram (Macrotyloma uniflorum) under drought stress. American Journal of Plant Physiology, 7: 17-29. 
10. Broadley, M.R., J. Alcock, J. Alford, P. Cartwright, I. Foot, S.J. Fairweather-Tait, D.J. Hart, R. Hurst, P. Knott and S.P. Mcgrath. 2010. Selenium biofortification of high-yielding winter wheat (Triticum aestivum L.) by liquid or granular Se fertilisation. Plant and Soil, 332(2-1): 5-18.

11. Danehlouipour, N. 2001. Investigation on cold resistance in rapeseed using quantitative characters and molecular markers. MSc. Thesis, Faculty of Agriculture, University of Tabriz, Tabriz, Iran.

12. Djanaguiraman, M., D.D. Devi, A.K. Shanker, J.A. Sheeba and U. Bangarusamy. 2005. Selenium-an antioxidative protectant in soybean during senescence. Plant and Soil, 272(1-2): 77-86.

13. Ducsay, L. and O. Lozek. 2006. Effect of selenium foliar application on its content in winter wheat grain. Plant, Soil and Environment, 52(2):78.

14. Ehteshami, S., A.A. Tehrani and B. Samadi. 2016. Effect of planting date on some phenological and morphological characteristics, yield and yield components of five rapeseed (Brassica napus L.) cultivars. Agronomy Journal, 108: 111-120 (In Persian).

15. Fallah-Haki, M.H., A. Yadavi, M. Movahhedi-Dehnavi, H. Balouchi and H. Faraji. 2012. The effect of planting date on phonological stages and quantity traits of four winter rapeseed cultivars in Yasouj. Journal of Plant Productions (Agronomy, Breeding and Horticulture), 35(2): 99-113 (In Persian).

16. Fang, Y., L. Wang, Z. Xin, L. Zhao, X. An and Q. Hu. 2008. Effect of foliar application of zinc, selenium, and iron fertilizers on nutrients concentration and yield of rice grain in China. Journal of Agricultural and Food Chemistry, 56(6): 2079-2084.

17. FAOSTAT. 2017. Agriculture organization of the united Nations statistics division (2019). Available in: http://www.fao.org/faostat/en/\#data.

18. Faraji, A. 2009. Estimating of planting and supplemental irrigation on dry matter distribution in canola cultivars (Brassica napus L.). Journal of Crop Breeding, 1(1): 29-42 (In Persian).

19. Faraji, A., N. Latifi, A. Soltani and A.H. Shirani-Rad. 2009. Seed yield and water use efficiency of canola (Brassica napus L.) as affected by high temperature stress and supplemental irrigation. Agricultural Water Management, 96(1): 132-140 (In Persian).

20. Farkhondeh, R., E. Nabizadeh and N. Jalilnezhad. 2012. Effect of salinity stress on proline content, membrane stability and water relations in two sugar beet cultivars. International Journal of AgriScience, 2(5): 385-392.

21. Fathi, G., S. Siadat and S. Hemaiaty. 2003. Effect of sowing date on yield and yield components of three oilseed rape varieties. Acta Agronomica Hungarica, 51(3): 249-255.

22. Filek, M., R. Keskinen, H. Hartikainen, I. Szarejko, A. Janiak, Z. Miszalski and A. Golda. 2008. The protective role of selenium in rape seedlings subjected to cadmium stress. Journal of Plant Physiology, 165(8): 833-844.

23. Germ, M., V. Stibilj, J. Osvald and I. Kreft. 2007. Effect of selenium foliar application on chicory (Cichorium intybus L.). Journal of Agricultural and Food Chemistry, 55(3): 795-798.

24. Gissel-Nielsen, G. 1981. Foliar application of selenite to barley plants low in selenium. Communications in Soil Science and Plant Analysis, 12(6): 631-642.

25. Grubbs, F.E. 1969. Procedures for detecting outlying observations in samples. Technometrics, 11(1): $1-21$.

26. Hajiboland, R., N. Keyvanfar, A .Joudmand, H. Rezae and M. Yousefnejad. 2015. Effect of selenium treatment on drought tolerance of canola plants. Journal of Plant Researches, 27(4):557-568 (In Persian).

27. Hartikainen, H., T. Xue and V. Piironen. 2000. Selenium as an anti-oxidant and pro-oxidant in ryegrass. Plant and Soil, 225(1-2): 193-200.

28. Hasanuzzaman, M., M.A. Hossain and M. Fujita. 2010. Selenium in higher plants: physiological role, antioxidant metabolism and abiotic stress tolerance. Journal of Plant Sciences, 5(4): 354-375.

29. Hashem, H.A ,.R.A. Hassanein, M.A. Bekheta and F.A. El-Kady. 2013. Protective role of selenium in canola (Brassica napus L.) plant subjected to salt stress. The Egyptian Journal of Experimental Biology (Botany), 9(2): 199-211.

30. Hu, Q., L. Chen, J. Xu, Y. Zhang and G. Pan. 2002. Determination of selenium concentration in rice and the effect of foliar application of Se-enriched fertiliser or sodium selenite on the selenium content of rice. Journal of the Science of Food and Agriculture, 82(8): 869-872.

31. Hu, Q., J. Xu and G. Pang. 2003. Effect of selenium on the yield and quality of green tea leaves harvested in early spring. Journal of Agricultural and Food Chemistry, 51(11): 3379-3381.

32. IRIMO. 2016. Iranian Meteorological Office Data Processing Center. Islamic Republic of Iran Meteorological Office.

33. Jiang, Y. and B. Huang. 2001. Effects of calcium on antioxidant activities and water relations associated with heat tolerance in two cool-season grasses. Journal of Experimental Botany, 52(355): 341-349.

34. Khan, M.A., M. Ashraf, S. Mujtaba, M. Shirazi, M. Khan, A. Shereen, S. Mumtaz, M.A. Siddiqui and G.M. Kaleri. 2010. Evaluation of high yielding canola type Brassica genotypes/mutants for drought tolerance using physiological indices as screening tool. Pakistan Journal of Botany, 42(6): 3807-3816.

35. Klessig, D.F. and J. Malamy. 1994. The salicylic acid signal in plants. Plant Molecular Biology, 26(5): 1439-1458.

36. Kumar, A. and D. Singh. 1998. Use of physiological indices as a screening technique for drought tolerance in oilseed Brassica species. Annals of Botany, 81(3): 413-420.

37. Kuznetsov, V.V., V. Kholodova, V.V. Kuznetsov and B. Yagodin. 2003. Selenium regulates the water status of plants exposed to drought. Doklady Biological Sciences, 390(1): 266-268. 
38. Larcher, W. and G. Neuner. 1989. Cold-induced sudden reversible lowering of in vivo chlorophyll fluorescence after saturating light pulses: a sensitive marker for chilling susceptibility. Plant Physiology, 89(3): 740-742.

39. Lazcano-Ferrat, I. and C.J. Lovatt. 1999. Relationship between relative water content, nitrogen pools, and growth of Phaseolus vulgaris L. and P. acutifolius A. Gray during water deficit. Crop Science, 39(2): 467-475.

40. Limin, A. and D. Fowler. 2000. Morphological and cytological characters associated with lowtemperature tolerance in wheat (Triticum aestivum L. em Thell.). Canadian Journal of Plant Science, 80(4): 687-692.

41. Lyons, G.H., Y. Genc, K. Soole, J. Stangoulis, F. Liu and R. Graham. 2009. Selenium increases seed production in Brassica. Plant and Soil, 318(1-2): 73-80.

42. Majidi, M.M., M. Jafarzadeh Ghahdarijani, F. Rashidi and A. Mirlohi. 2016. Relationship of different traits in rapeseed (Brassica napus L.) cultivars under normal and drought conditions. Journal of Crop Breeding, 8(17): 55-65 (In Persian).

43. Miller, P.R., B.G. Mcconkey, G.W. Clayton, S.A. Brandt, J.A. Staricka, A.M. Johnston, G.P. Lafond, B.G. Schatz, D.D. Baltensperger and K.E. Neill. 2002. Pulse crop adaptation in the northern Great Plains. Agronomy Journal, 94(2): 261-272.

44. Mostafavi-Rad, M. and A. Mirabdolhaq. 2010. Evaluation of delayed sowing dates on quantitative and qualitative traits and dry matter remobilization in three winter rapeseed cultivars in Markazi Province. Plant Production, 33(1): 49-66 (In Persian).

45. Munns, R. and M. Tester. 2008. Mechanisms of salinity tolerance. Annual Review of Plant Biology, 59: 651-681.

46. Naji, A.M. 2001. Evaluation of cold resistance in oilseed rape genotypes. M.Sc. Thesis ,Tabriz Univeisity, 117 pp (In Persian).

47. Nawaz, F., R. Ahmad, M. Ashraf, E. Waraich and S. Khan. 2015. Effect of selenium foliar spray on physiological and biochemical processes and chemical constituents of wheat under drought stress. Ecotoxicology and Environmental Safety, 113: 191-200.

48. Nejat, F., M. Dadniya, M. Shirzadi and S .Lak. 2009. Effects of drought stress and selenium application on yield and yield components of two maize cultivars. Plant Ecophysiology, 2: 95-102 (In Persian).

49. Nowak, J., K. Kaklewski and M. Ligocki. 2004. Influence of selenium on oxidoreductive enzymes activity in soil and in plants. Soil Biology and Biochemistry, 36(10): 1553-1558.

50. Ozer, H. 2003. Sowing date and nitrogen rate effects on growth, yield and yield components of two summer rapeseed cultivars. European Journal of Agronomy, 19(3): 453-463.

51. Pasban-Eslam, B. 2011. Study of possibility of delayed planting of oilseed rape (Brassica napus L.) in East Azarbaijan in Iran. Seed and Plant Production, 27(3): 269-284 (In Persian).

52. Pasban-Eslam, B., M. Shakiba, M. Neyshabouri, M. Moghadam and M. Ahmadi. 2000. Evaluation of physiological indices as a screening technique for drought resistance in oilseed rape. Pakistan Academy of Sciences Journal, 37: 143-152.

53. Poggi, V., A. Arcioni, P. Filippini and P.G. Pifferi. 2000. Foliar application of selenite and selenate to potato (Solanum tuberosum): Effect of a ligand agent on selenium content of tubers. Journal of Agricultural and Food Chemistry, 48(10): 4749-4751.

54. Prášil, I., Z. Kadlecová-Faltusová and M. Faltus .2001. Water and ABA content in fully expanded leaves of cold-hardened barleys. Icelandic Agricultural Sciences, 14: 49-53.

55. Raymer, P. 2002. Canola: an emerging oilseed crop. Trends in New Crops, 1: 122-126.

56. Robertson, M. and J. Holland. 2004. Production risk of canola in the semi-arid subtropics of Australia. Australian Journal of Agricultural Research, 55(5): 525-538.

57. Saidi, I., Y. Chtourou and W. Djebali. 2014. Selenium alleviates cadmium toxicity by preventing oxidative stress in sunflower (Helianthus annuus) seedlings. Journal of Plant Physiology, 171(5): 8591.

58. Sajedi, N.A., M.R. Ardakani, H. Madani, A. Naderi and M. Miransari. 2011. The effects of selenium and other micronutrients on the antioxidant activities and yield of corn (Zea mays L.) under drought stress. Physiology and Molecular Biology of Plants, 17(3): 215-222.

59. SAS. 2008. SAS/STAT User's Guide Version 9.1: SAS Institute, Cary, NC.

60. Seppänen, M., M. Turakainen and H. Hartikainen. 2003. Selenium effects on oxidative stress in potato. Plant Science, 165(2): 311-319.

61. Seppänen, M.M., J. Kontturi, I.L. Heras, Y. Madrid, C. Cámara and H. Hartikainen. 2010. Agronomic biofortification of Brassica with selenium-enrichment of SeMet and its identification in Brassica seeds and meal. Plant and Soil, 337(1-2): 273-283.

62. Shapiro, S.S. and M.B. Wilk. 1965. An analysis of variance test for normality (complete samples). Biometrika, 52(3/4): 591-611.

63. Sharma, S., A. Bansal, S.K. Dhillon and K.S. Dhillon. 2010. Comparative effects of selenate and selenite on growth and biochemical composition of rapeseed (Brassica napus L.). Plant and Soil, 329(1-2): 339-348.

64. Shedeed, S.I., Z.F. Fawzy and A.E.M. Mahmoud. 2018. Nano and mineral selenium foliar application effect on pea plants (Pisum sativum L.). Bioscience Research, 15(2): 645-654. 
65. Shirani-Rad, A., Z. Bitarafan, F. Rahmani, T. Taherkhani, A. Moradi-Aghdam and S. Nasr-Esfahani. 2014. Evaluation of spring rapeseed (Brassica napus L.) cultivars for different planting dates and irrigation regimes. Journal of Animal and Plant Sciences, 24(4): 1166-1172.

66. Shirani-Rad, A., N .Shahsavari, H. Jais, A. Dadrasnia, A. Askari and M. Saltughi. 2015. Fall cultivar of rapeseed (Brassica napus) for reduction of damage due to late season drought. Indian Journal of Agricultural Sciences, 85(1): 50-54.

67. Smoleńska, G. and P.J. Kuiper. 1977. Effect of low temperature upon lipid and fatty acid composition of roots and leaves of winter rape plants. Physiologia Plantarum, 41(1): 29-35.

68. Szabados, L. and A. Savoure. 2010. Proline: a multifunctional amino acid. Trends in Plant Science, 15(2): 89-97.

69. Uzun, B. and S. Furat. 2009. Sowing date effects on growth, flowering, oil content and seed yield of canola cultivars. Asian Journal of Chemistry, 21(3): 1957-1965.

70. Valadabadi, S.A., A.H. Shirani-Rad and H.A. Farahani. 2010. Ecophysiological influences of zeolite and selenium on water deficit stress tolerance in different rapeseed cultivars. Journal of Ecology and the Natural Environment, 2(8): 154-159.

71. Verbruggen, N. and C. Hermans. 2008. Proline accumulation in plants: a review. Amino Acids, 35(4): 753-759.

72. Xue, T., H. Hartikainen and V. Piironen. 2001. Antioxidative and growth-promoting effect of selenium on senescing lettuce. Plant and Soil, 23(7): 55-61.

73. Yadav, R. and C. Bhushan. 2001. Effect of moisture stress on growth and yield in rice genotypes. Indian Journal of Agricultural Research, 35(2): 104-107.

74. Yang, F., L. Chen, Q. Hu and G. Pan. 2003. Effect of the application of selenium on selenium content of soybean and its products. Biological Trace Element Research, 93(1-3): 249-256.

75. Yao, X., C. Jianzhou, H. Xueli, L. Binbin, L. Jingmin and Y. Zhaowei. 2013. Effects of selenium on agronomical characters of winter wheat exposed to enhanced ultraviolet-B. Ecotoxicology and Environmental Safety, 92: 320-326.

76. Ylaranta, T. 1983. Effect of applied selenite and selenate on the selenium content of barley (Hordeum vulgare). Annales Agriculturae Fenniae, 22: 164-174.

77. Zahedi, H., A.H. Shirani-Rad and H.R. Tohidi-Moghadam. 2018. Effect of zeolite and selenium foliar application on growth, production and some physiological attributes of three canola (Brassica napus L.) cultivars subjected to drought stress. Revista Científica UDO Agrícola, 12(1): 135-142.

78. Zhu, Y.G., Y. Huang, Y. Hu, Y. Liu and P. Christie. 2004. Interactions between selenium and iodine uptake by spinach (Spinacia oleracea L.) in solution culture. Plant and Soil Biology, 261(1-2): 99105.

79. Zhu, Y.G., E.A. Pilon-Smits, F.J. Zhao, P.N. Williams and A. Meharg. 2009. Selenium in higher plants: understanding mechanisms for biofortification and phytoremediation. Trends in Plant Science, 14(8): 436-442. 


\title{
Effect of Selenium Foliar Application on Some Quantitative and Qualitative Characteristics of Rapeseed Cultivars under End-Season Thermal Stress
}

\author{
Abdoreza Davoudi ${ }^{1}$, Hossein Zeinalzadeh-Tabrizi ${ }^{2}$ and Amirhossein Shirani-Rad ${ }^{3}$ \\ 1- PhD, Department of Agronomy and Plant Breeding, Islamic Azad University, Tabriz Branch, Tabriz, Iran \\ 2- Assistant Professor, Crop and Horticultural Science Research Department, Ardabil Agricultural and Natural \\ Resources Research and Education Center, AREEO, Iran \\ (Corresponding author: h.zeinalzadeh@areeo.ac.ir) \\ 3- Professor, Seed and Plant Improvement Institute (SPII), Agricultural Research, Education and Extension \\ Organization (AREEO), Karaj, Iran \\ Received: August 27, $2019 \quad$ Accepted: October 15, 2019
}

\begin{abstract}
Selenium has a protective and positive effect on the quantitative and qualitative characteristics of the plants. In order to investigate the effect of selenium foliar application on leaf proline content and some quantitative and qualitative characteristics of rapeseed cultivars under end-season thermal stress, a factorial split plot experiment was conducted in a randomized complete block design with three replications for two years (2014-2016) in Karaj, Iran. In this experiment, the first factor was planting date on October 15 (timed planting), October 25 (relatively late planting) and November 5 (delayed planting), the second factor was selenium in two levels, including non-application and application of 30 grams per liter of sodium selenate and third factor was genotype, including a commercial cultivar, two promising lines and three foreign commercial hybrids, so that the first and second factor were factorially in the main plots and the third factor was split plot as in sub plot. The results showed that quantitative and qualitative characteristics of rapeseed in this experiment were affected by planting date. Delay in planting reduced winter survival, biological yield, seed yield, crown diameter, relative water content of leaves of rapeseed genotypes. Furthermore, the delay in planting caused a significant increase in the crown water and leaf proline content in the rosette stage, but did not have a significant effect on the harvest index of the genotypes. Selenium foliar application improved the winter survival, biological yield, seed yield, crown diameter and relative water content of leaves of rapeseed genotypes. Selenium foliar application also significantly reduced leaf proline content in the rosette stage, which was an important factor in tolerance of genotypes to delayed planting and end-season thermal stress. In terms of winter survival, seed and biological yield, the L72 line showed the best response to these traits in timed planting, but in terms of delayed planting, Opera was better than the other genotypes, although there was no significant difference with the L72 line.
\end{abstract}

Keywords: End-Season Thermal Stress, Planting Date, Proline, Rapeseed, Sodium Selenate 